LMU-TPW 00-20

\title{
Nonabelian noncommutative gauge theory via noncommutative extra dimensions
}

\author{
Branislav Jurčo, Peter Schupp and Julius Wess \\ Universität München, Sektion Physik \\ Theresienstr. 37, 80333 München, Germany
}

\begin{abstract}
The concept of covariant coordinates on noncommutative spaces leads directly to gauge theories with generalized noncommutative gauge fields of the type that arises in string theory with background $B$-fields. The theory is naturally expressed in terms of cochains in an appropriate cohomology; we discuss how it fits into the framework of projective modules. The equivalence of star products that arise from the background field with and without fluctuations and Kontsevich's formality theorem allow an explicitly construction of a map that relates ordinary gauge theory and noncommutative gauge theory (Seiberg-Witten map.) As application we show the exact equality of the Dirac-Born-Infeld action with $B$-field in the commutative setting and its semi-noncommutative cousin in the intermediate picture. Using noncommutative extra dimensions the construction is extended to noncommutative nonabelian gauge theory for arbitrary gauge groups; an explicit map between abelian and nonabelian gauge fields is given. All constructions are also valid for non-constant $B$-field, Poisson structure and metric.
\end{abstract}

e-mail: jurco,schupp,wess @ theorie.physik.uni-muenchen.de 


\section{Contents}

$\begin{array}{lll}1 & \text { Introduction } & \mathbf{2}\end{array}$

1.1 Noncommutative gauge theory in string theory . . . . . . . . . . . . . . . 3

\begin{tabular}{|ll|l}
2 & Gauge theory on noncommutative spaces & 5
\end{tabular}

2.1 Covariant functions, covariant coordinates . . . . . . . . . . . . 5

2.1 .1 Canonical structure (constant $\theta$ ) and noncommutative Yang-Mills 6

2.2 Abstract formulation of noncommutative gauge theory . . . . . . . . . . 7

2.2 .1 Projective modules . . . . . . . . . . . . . . . . . 10

$\begin{array}{lll}3 & \text { Ordinary versus noncommutative gauge theory } & 11\end{array}$

3.1 Motivation from string theory . . . . . . . . . . . . . . . . . . . . 12

3.2 Quantum version of Moser's lemma: Seiberg-Witten map . . . . . . . . . 13

3.2.1 Semi-classical construction . . . . . . . . . . . . . . . . . 14

3.2 .2 Kontsevich formality map . . . . . . . . . . . . . . . . . . . . 16

3.2.3 Quantum construction . . . . . . . . . . . . . . . . . . . 18

3.3 Covariance and (non)uniqueness . . . . . . . . . . . . . . . . . . . 20

3.4 Dirac-Born-Infeld action in the intermediate picture . . . . . . . . . . . . 21

3.5 Some notes on symmetric tensors . . . . . . . . . . . . . . . . . . 25

$\begin{array}{lll}4 & \text { Seiberg-Witten map for nonabelian gauge fields } & 27\end{array}$

4.1 Nonabelian setting . . . . . . . . . . . . . . . . . . . . 27

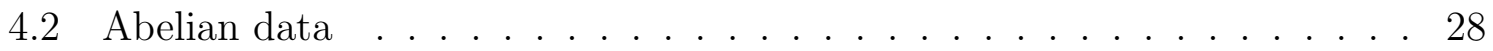

$4.3 \quad$ Mini Seiberg-Witten map . . . . . . . . . . . . . . . . . . . . . 31

\begin{tabular}{|l|l|}
\hline A Brackets, evolution and parameters & 32
\end{tabular}

A.1 Schouten-Nijenhuis bracket . . . . . . . . . . . . . . . . . . . . . 32

A.2 Gerstenhaber bracket . . . . . . . . . . . . . . . . . . . . 33

A.3 t-evolution . . . . . . . . . . . . . . . . . . . . . . . . . . . . . 33

A.4 Semi-classical and quantum gauge parameters . . . . . . . . . . . . . . 34

\begin{tabular}{ll}
\hline References & 35
\end{tabular} 


\section{Introduction}

A natural approach to gauge theory on noncommutative spaces can be based on the simple observation that multiplication of a field by a (coordinate) function is not a covariant concept if that function does not commute with gauge transformations [1]. This can be cured by adding appropriate noncommutative gauge potentials and thus introducing covariant functions in complete analogy to the covariant derivatives of ordinary gauge theory. This construction is of particular interest because of its apparent relevance for the description of open strings in a background $B$-field [2, 3, 4, where the D-brane world volume can be interpreted as a noncommutative space whose fluctuations are governed by a noncommutative version of Yang-Mills theory [5, 6, 7, 8, 9. It has been noticed (at least in the case of a constant $B$-field) that there can be equivalent description of the effective theory both in terms of noncommutative gauge theory and ordinary gauge theory. From the physics perspective the two pictures are related by a choice of regularization [9, 10] which suggests a somewhat miraculous field redefinition that is usually called Seiberg-Witten map [9]. The inverse $B$-field, or more generally the antisymmetric part of the inverse sum of $B$-field and metric, defines a Poisson structure $\theta$ whose quantization gives rise to the noncommutativity on the D-brane world volume. Classically, the field strength $f$ describes fluctuations of the $B$-field. The Seiberg-Witten map expresses the noncommutative potential $\hat{A}$, noncommutative gauge parameter $\hat{\lambda}$ and noncommutative field strength in terms of their classical counter parts $a, \lambda, f$ as formal power series in $\theta$, such that noncommutative gauge transformations $\hat{\delta}_{\hat{\lambda}}$ are induced by ordinary gauge transformations $\delta_{\lambda}$ :

$$
\hat{A}(a)+\hat{\delta}_{\hat{\lambda}} \hat{A}(a)=\hat{A}\left(a+\delta_{\lambda} a\right),
$$

where $\hat{\lambda}$ is a function both of $a$ and $\lambda$. In previous work 11, 12 we have focused on the rank one case and have explicitly constructed the maps $\hat{A}(a)$ and $\hat{\lambda}(a, \lambda)$ to all orders in theta for the general case of an arbitrary Poisson manifolds which are relevant for the case of non-constant background fields. 2] The corresponding star products can be computed with Kontsevich's formula [13]; this formula continues to make sense even for non-closed $B$-field although the corresponding star product will no longer be associative (see also [14]) but the non-associativity is still under control by the formality (90). For a noncommutative gauge theory the rank one case does already include some information about the nonabelian case, since it is always possible to include a matrix factor in the definition of the underlying noncommutative space. In this article we shall make this more precise and will extend our previous results to non-abelian

\footnotetext{
${ }^{1}$ From the phase space point of view ordinary gauge theory is in fact a special case of this construction with gauge potentials for only half of the 'coordinates' (momenta).

${ }^{2}$ This is of course neither restricted to magnetic fields $-B^{0 i}$ need not be zero - nor to even dimensional manifolds.
} 
gauge theories for any Lie group. (A brief description of the construction was given in [15].) The noncommutative gauge potential and field strength in general take values in the universal enveloping algebra, nevertheless thanks to the existence of the SeibergWitten map the theory can be consistently formulated in terms of only a finite number of fields; this important observation has been discussed in [16]. A prerequisite for all this is an appropriate formulation of gauge theory on a more or less arbitrary noncommutative space. (Here we are interested in the general case of an arbitrary associative algebra of non-commuting variables, important special examples with constant, linear and quadratic commutation relations have been discussed in [1].) Particularly wellsuited is the approach based on the notion of covariant coordinates that we mentioned above because it finds a natural interpretation in the framework of deformation quantization [17, 13, 18. This is the natural setting since we are dealing with associative algebras and formal power series - it also allows rigorous statements by postponeing, or rather circumventing difficult questions related to convergence and representation theory. Deformation quantization for non-constant and possibly degenerate Poisson structures goes far beyond the basic Weyl-Moyal product and the problem has only recently found a general solution [13]. To construct a Seiberg-Witten map we do in fact need the even more general formality theorem of Kontsevich [13]. A link between Kontsevich quantization/formality and quantum field theory is given by the path integral approach 19] which relates the graphs that determine the terms in the formality map to Feynman diagrams. The relevant action - a Poisson sigma model - was originally studied in [20, 21]; see also [22, 23]. Our discussion is entirely tree-level. Aspects of the quantization of nonabelian noncommutative gauge theories have been discussed by several authors [24, 25, 26] (and references therein.) Closest to the present discussion is the perturbative study of $\theta$-expanded noncommutative gauge theories [27, 28].

\subsection{Noncommutative gauge theory in string theory}

Let us briefly recall how star products and noncommutative gauge theory arise in string theory [3, 4, 9]: Consider an open string $\sigma$-model with background term

$$
S_{B}=\frac{1}{2 i} \int_{D} B_{i j} \epsilon^{a b} \partial_{a} x^{i} \partial_{b} x^{j},
$$

where the integral is over the string world-sheet (disk) and $B$ is constant, nondegenerate and $d B=0$. The correlation functions on the boundary of the disc in the decoupling limit $\left(g \rightarrow 0, \alpha^{\prime} \rightarrow 0\right)$ are

$$
\left\langle f_{1}\left(x\left(\tau_{1}\right)\right) \cdot \ldots \cdot f_{n}\left(x\left(\tau_{n}\right)\right)\right\rangle_{B}=\int d x f_{1} \star \ldots \star f_{n}, \quad\left(\tau_{1}<\ldots<\tau_{n}\right)
$$

with the Weyl-Moyal star product

$$
(f \star g)(x)=\left.e^{\frac{i \hbar}{2} \theta^{i j} \partial_{i} \partial_{j}^{\prime}} f(x) g\left(x^{\prime}\right)\right|_{x^{\prime} \rightarrow x},
$$


which is the deformation quantization of the Poisson structure $\theta=B^{-1}$. More generally a star product is an associative, $[[\hbar]]$-bilinear product

$$
f \star g=f g+\sum_{n=1}^{\infty}(i \hbar)^{n} \underbrace{B_{n}(f, g)}_{\text {bilinear }},
$$

which is the deformation of a Poisson structure $\theta$ :

$$
[f \stackrel{\star}{,} g]=i \hbar\{f, g\}+\mathcal{O}\left(\hbar^{2}\right), \quad\{f, g\}=\theta^{i j}(x) \partial_{i} f \partial_{j} g .
$$

We now perturb the constant $B$ field by adding a gauge potential $a_{i}(x): B \rightarrow B+d a$, $S_{B} \rightarrow S_{B}+S_{a}$, with

$$
S_{a}=-i \int_{\partial D} d \tau a_{i}(x(\tau)) \partial_{\tau} x^{i}(\tau)
$$

Classically we have the naive gauge invariance

$$
\delta a_{i}=\partial_{i} \lambda
$$

but in the quantum theory this depends on the choice of regularization. For PauliVillars (8) remains a symmetry but if one expands $\exp S_{a}$ and employes a point-splitting regularization then the functional integral is invariant under noncommutative gauge transformations

$$
\hat{\delta} \hat{A}_{i}=\partial_{i} \hat{\lambda}+i \hat{\lambda} \star \hat{A}_{i}-i \hat{A}_{i} \star \hat{\lambda}
$$

Since a sensible quantum theory should be independent of the choice of regularization there should be field redefinitions $\hat{A}(a), \hat{\lambda}(a, \lambda)$ (Seiberg-Witten map) that relate (8) and (9):

$$
\hat{A}(a)+\hat{\delta}_{\hat{\lambda}} \hat{A}(a)=\hat{A}\left(a+\delta_{\lambda} a\right) .
$$

It is instructive to study the effect of the extra factor $\exp S_{a}$ in the correlation function (3) in more detail: It effectively shifts the coordinatest

$$
x^{i} \rightarrow x^{i}+\theta^{i j} \hat{A}_{j}=: \mathcal{D} x^{i} .
$$

More generally, for a function $f$,

$$
f \rightarrow f+f_{A}=: \mathcal{D} f .
$$

\footnotetext{
${ }^{3}$ In this form this formula is only valid for the Moyal-Weyl star product.

${ }^{4}$ Notation: $\mathcal{D}$ should not be confused with a covariant derivative (but it is related).
} 
The mapping $\mathcal{A}: f \mapsto f_{A}$ plays the role of a generalized gauge potential; it maps a function to a new function that depends on the gauge potential. The shifted coordinates and functions are covariant under noncommutative gauge transformations:

$$
\hat{\delta}\left(\mathcal{D} x^{i}\right)=i\left[\hat{\lambda} * \mathcal{D} x^{i}\right], \quad \hat{\delta}(\mathcal{D} f)=i[\hat{\lambda} \stackrel{\star}{ }, \mathcal{D} f] .
$$

The first expression implies (9) (for $\theta$ constant and nondegenerate).

The covariant coordinates (11) are the background independent operators of [9, 32]; they and the covariant functions (12) can also be introduced abstractly in the general case of an arbitrary noncommutative space as we shall discuss in the next section.

\section{Gauge theory on noncommutative spaces}

\subsection{Covariant functions, covariant coordinates}

Take a more or less arbitrary noncommutative space, i.e. an associative unital algebra $\mathcal{A}_{x}$ of noncommuting variables with multiplication $\star$ and consider (matter) fields $\psi$ on this space. The fields can be taken to be elements of $\mathcal{A}_{x}$, or, more generally, a left module of it. The notion of a gauge transformation is introduced as usual p

$$
\psi \mapsto \Lambda \star \psi
$$

where $\Lambda$ is an invertible element of $\mathcal{A}_{x}$. In analogy to commutative geometry where a manifold can be described by the commutative space of functions over it, we shall refer to the elements of $\mathcal{A}_{x}$ also as functions. Later we shall focus on the case where the noncommutative multiplication is a star product; the elements of $\mathcal{A}_{x}$ are then in fact ordinary functions in the usual sense of the word. The left-multiplication of a field with a function $f \in \mathcal{A}_{x}$ does in general not result in a covariant object because of the noncommutativity of $\mathcal{A}_{x}$ :

$$
f \star \psi \mapsto f \star \Lambda \star \psi \neq \Lambda \star(f \star \psi) .
$$

(As in ordinary gauge theory the gauge transformation only acts on the fields, i.e. on the elements of the left-module of $\mathcal{A}_{x}$ and not on the elements of $\mathcal{A}_{x}$ itself.) To cure (15) we introduce covariant functions

$$
\mathcal{D} f=f+f_{A},
$$

that transform under gauge transformations by conjugation

$$
\mathcal{D} f \mapsto \Lambda \star \mathcal{D} f \star \Lambda^{-1},
$$

\footnotetext{
${ }^{5}$ We shall often use the infinitesimal version $\delta \psi=i \lambda \star \psi$ of (14) - this is purely for notational clarity. Other transformations, like, e.g., $\psi \mapsto \psi \star \Lambda$ or $\psi \mapsto \Lambda \star \psi \star \Lambda^{-1}$ can also be considered.
} 
by adding 'gauge potentials' $f_{A}$ with appropriate transformation property

$$
f_{A} \mapsto \Lambda \star\left[f^{\star}, \Lambda^{-1}\right]+\Lambda \star f_{A} \star \Lambda^{-1} .
$$

Further covariant objects can be constructed from covariant functions; the '2-tensor'

$$
\mathcal{F}(f, g)=[\mathcal{D} f \star, \mathcal{D} g]-\mathcal{D}([f \star, g])
$$

for instance plays the role of covariant noncommutative field strength.

\subsubsection{Canonical structure (constant $\theta$ ) and noncommutative Yang-Mills}

Before we continue let us illustrate all this in the particular simple case of an algebra $\mathcal{A}_{x}$ generated by 'coordinates' $x^{i}$ with canonical commutation relations

$$
\left[x^{i} \stackrel{\star}{,} x^{j}\right]=i \theta^{i j}, \quad \theta^{i j} \in \mathbb{C} .
$$

This algebra arises in the decoupling limit of open strings in the presence of a constant $B$-field. It can be viewed as the quantization of a Poisson structure with Poisson tensor $\theta^{i j}$ and the multiplication $\star$ is then the Weyl-Moyal star product

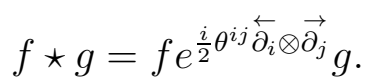

(This formula holds only in the present example, where $\theta^{i j}$ is constant and we shall also assume that it is non-degenerate. In the rest of the paper we drop both restrictions.)

Let us focus on the coordinate functions $x^{i}$. The corresponding covariant coordinates are

$$
\mathcal{D} x^{i}=x^{i}+x_{A}^{i}=x^{i}+\theta^{i j} \hat{A}_{j},
$$

where we have used $\theta$ to lower the index on $\hat{A}_{j}$. Using (20), we see that the transformation (18) of the noncommutative gauge potential $\hat{A}_{j}$ is

$$
\hat{A}_{j} \mapsto i \Lambda \star \partial_{j}\left(\Lambda^{-1}\right)+\Lambda \star \hat{A}_{j} \star \Lambda^{-1},
$$

or, infinitesimally

$$
\delta \hat{A}_{j}=\partial_{j} \lambda+i\left[\lambda, \hat{A}_{j}\right]_{\star} .
$$

The noncommutative field strength

$$
\hat{F}_{k l}=\partial_{k} \hat{A}_{l}-\partial_{l} \hat{A}_{k}-i\left[\hat{A}_{k}, \hat{A}_{l}\right]_{\star}
$$

\footnotetext{
${ }^{6}$ Notation: $[a \stackrel{\star}{,} b] \equiv a \star b-b \star a \equiv[a, b]_{\star}$.
} 
transforms covariantly

$$
\hat{F}_{k l} \mapsto \Lambda \star \hat{F}_{k l} \star \Lambda^{-1} .
$$

We have again used $\theta$ to lower indices to get (25) from the definition (19)

$$
i \hat{F}_{k l} \theta^{i k} \theta^{j l} \equiv \mathcal{F}\left(x^{i}, x^{j}\right)=\left[x_{A}^{i}, x^{j}\right]_{\star}+\left[x^{i}, x_{A}^{j}\right]_{\star}+\left[x_{A}^{i}, x_{A}^{j}\right]_{\star} .
$$

Note, that we should in general be more careful when using $\theta$ to lower indices as in (22) or (27) because this may spoil the covariance when $\theta$ is not constant as it was in this particular example. Relations (23), (25) and (26) define what is usually called Noncommutative Yang-Mills theory (NCYM) in the narrow sense: ordinary Yang-Mills with all matrix products replaced by star products. This simple rule, however, only really works well for the Moyal-Weyl product, i.e. constant $\theta$. In the general case it is wise to stick with the manifestly covariant and coordinate-independent] objects defined in (16) and (19). The fundamental objects are really the mappings (differential operators) $\mathcal{D}$ and $\mathcal{F}$ in these equations. The transformation of $\mathcal{A}=\mathcal{D}-\mathrm{id}: f \mapsto f_{A}$ under gauge transformations is exactly so that (16) transforms by conjugation. The mappings $\mathcal{A} \in \operatorname{Hom}\left(\mathcal{A}_{x}, \mathcal{A}_{x}\right)$ and $\mathcal{F} \in \operatorname{Hom}\left(\mathcal{A}_{x} \wedge \mathcal{A}_{x}, \mathcal{A}_{x}\right)$ play the role of generalized noncommutative gauge potential and noncommutative field strength. There are several reasons, why one needs $\mathcal{A}$ and $\mathcal{D}$ and not just $A^{i} \equiv \mathcal{A}\left(x^{i}\right)$ (or $\hat{A}_{i}$, for $\theta$ constant): If we perform a general coordinate transformation $x^{i} \mapsto x^{i^{\prime}}\left(x^{j}\right)$ and transform $A^{i}$ (or $\hat{A}_{i}$ ) naively as its index structure suggests, then we would obtain objects that are no longer covariant under noncommutative gauge transformations. The correct transformation, $\mathcal{A}\left(x^{i}\right) \mapsto \mathcal{A}^{\prime}\left(x^{i^{\prime}}\right)$, is more complicated and will be discussed in section 3.3. Furthermore we may be interested in covariant versions of scalar fields $\phi(x)$. These are given by the corresponding covariant function $\mathcal{D}(\phi(x))$.

In the next section we will propose an abstract definition of the type of noncommutative gauge theory that is of present interest. Then we shall proceed to give an interpretation in the framework of deformation quantization and will construct a particular important class of these operators.

\subsection{Abstract formulation of noncommutative gauge theory}

Finite projective modules take the place of fiber bundles in the noncommutative realm [29]. This is also the case here, as we shall explain below, but may not have been apparent since we have been working with component fields as is customary in the physics literature. We have argued in the previous section that $\mathcal{A} \in C^{1}, \mathcal{F} \in C^{2}$ with $C^{p}=\operatorname{Hom}\left(\mathcal{A}_{x}^{\wedge p}, \mathcal{A}_{x}\right), C^{0} \equiv \mathcal{A}_{x}$. These $p$-cochains take the place of forms on a noncommutative space $\mathcal{A}_{x}$, which for now is still an arbitrary associative algebra over a

\footnotetext{
${ }^{7}$ We would like to thank Anton Alekseev for stressing the importance of this point.
} 
field $k$ with multiplication $\star$. It is actually more convenient to start with the Hochschild complex of $\mathcal{A}_{x}, H^{p}\left(\mathcal{A}_{x}, \mathcal{A}_{x}\right)=\operatorname{Hom}_{k}\left(\mathcal{A}_{x}^{\otimes p}, \mathcal{A}_{x}\right)$, with values in $\mathcal{A}_{x}$ considered as a left module of $\mathcal{A}_{x}$. (The formulas for $C^{p}$ can then be obtained by antisymmetrization.) We have a coboundary operator $\mathbf{d}_{\star}: H^{p} \rightarrow H^{p+1}, \mathbf{d}_{\star}^{2}=0, \mathbf{d}_{\star} 1=0$,

$$
\mathbf{d}_{\star} \mathcal{C}=-[\mathcal{C}, \star]_{\mathrm{G}},
$$

where $[,]_{\mathrm{G}}$ is the Gerstenhaber bracket (A.4),

$$
\begin{aligned}
\left(\mathbf{d}_{\star} \mathcal{C}\right) & \left(f_{1}, \ldots, f_{p+1}\right) \\
= & f_{1} \star \mathcal{C}\left(f_{2}, \ldots, f_{p+1}\right)-\mathcal{C}\left(f_{1} \star f_{2}, f_{3}, \ldots, f_{p+1}\right)+\mathcal{C}\left(f_{1}, f_{2} \star f_{3}, \ldots, f_{p+1}\right) \\
& \mp \cdots+(-)^{p} \mathcal{C}\left(f_{1}, f_{2}, \ldots, f_{p} \star f_{p+1}\right)+(-)^{p+1} \mathcal{C}\left(f_{1}, \ldots, f_{p}\right) \star f_{p+1}
\end{aligned}
$$

and we have a cup product $\star: H^{p_{1}} \otimes H^{p_{2}} \rightarrow H^{p_{1}+p_{2}}$,

$$
\left(\mathcal{C}_{1} \star \mathcal{C}_{2}\right)\left(f_{1}, \ldots, f_{p_{1}+p_{2}}\right)=\mathcal{C}_{1}\left(f_{1}, \ldots, f_{p_{1}}\right) \star \mathcal{C}_{2}\left(f_{p_{1}+1}, \ldots, f_{p_{1}+p_{2}}\right) .
$$

For a function $\lambda \in H^{0} \equiv \mathcal{A}_{x}$ the coboundary operator is defined as

$$
\left(\mathbf{d}_{\star} \lambda\right)(f)=f \star \lambda-\lambda \star f
$$

and the cup product reduces to the multiplication $\star$ of $\mathcal{A}_{x}$ in the obvious way. For this reason and since there seems to be little chance of confusion we have used the same symbol $\star$ for the cup product and the multiplication. Let us apply the Hochschild formalism to the gauge transformation dependent map $\mathcal{D} \in H^{1}$ that we introduced in the definition of covariant functions (16) in the previous section. In view of the way that $A^{i} \equiv \theta^{i j} \hat{A}_{j}$ appeared in the definition of covariant coordinates (22) we define an abstract noncommutative gauge potential $\mathcal{A} \in H^{1}$

$$
\mathcal{A} \equiv \mathcal{D}-\mathrm{id}
$$

Applying $\mathcal{A}$ to coordinate functions in the setting of (22) we indeed recover $A^{i}$ :

$$
\mathcal{A}\left(x^{i}\right)=\mathcal{D}\left(x^{i}\right)-x^{i}=A^{i} .
$$

Let us compute the behavior of $\mathcal{A}$ under a gauge transformation. Using (17) and the definitions of $\mathbf{d}_{\star}$ and the cup product we find

$$
\mathcal{A} \mapsto \Lambda \star \mathrm{d}_{\star} \Lambda^{-1}+\Lambda \star \mathcal{A} \star \Lambda^{-1},
$$

which gives (18) when evaluated on a function. The corresponding infinitesimal version is

$$
\delta \mathcal{A}=i\left(-\mathbf{d}_{\star} \lambda+\lambda \star \mathcal{A}-\mathcal{A} \star \lambda\right) .
$$


Next we introduce the "Hochschild" field strength $\mathcal{F}_{H} \in H^{2}$

$$
\mathcal{F}_{H} \equiv \mathrm{d}_{\star} \mathcal{A}+\mathcal{A} \star \mathcal{A}
$$

and compute

$$
\mathcal{F}_{H}(f, g)=\mathcal{D} f \star \mathcal{D} g-\mathcal{D}(f \star g)
$$

and find the Bianchi identity

$$
\mathrm{d}_{\star} \mathcal{F}_{H}+\mathcal{A} \star \mathcal{F}_{H}-\mathcal{F}_{H} \star \mathcal{A}=0 .
$$

Evaluated on three functions $f, g, h$ the latter reads

$$
\mathcal{D}((f \star g) \star h)-\mathcal{D}(f \star(g \star h))+(\mathcal{D} f \star \mathcal{D} g) \star \mathcal{D} h-\mathcal{D} f \star(\mathcal{D} g \star \mathcal{D} h),
$$

which is zero by associativity of $\mathcal{A}_{x} . \mathcal{F}_{H}$ transforms covariantly under gauge transformations

$$
\mathcal{F}_{H} \mapsto \Lambda \star \mathcal{F}_{H} \star \Lambda^{-1}
$$

infinitesimally

$$
\delta \mathcal{F}_{H}=i\left(\lambda \star \mathcal{F}_{H}-\mathcal{F}_{H} \star \lambda\right) .
$$

When we compare (19) and (36), we see (as expected) that our noncommutative field strength $\mathcal{F}$ of the previous section is an antisymmetric version of $\mathcal{F}_{H}$. We can obtain $\mathcal{F}$ directly by taking the antisymmetrized version of Hochschild, where one considers $\mathcal{A}_{x}$ as a Lie algebra with bracket $[a \star b]=a \star b-b \star a$; this is the Chevalley cohomology of $\mathcal{A}_{x}$ with values in $\mathcal{A}_{x}: C^{p}=\operatorname{Hom}_{k}\left(\mathcal{A}_{x}^{\wedge p}, \mathcal{A}_{x}\right)$. We find the relevant formulas in this setting by replacing $H^{p}$ with $C^{p}$ (whose elements are antisymmetric), and by using corresponding antisymmetrized formulas for the coboundary operator $\mathbf{d}_{\star}$ and the cup product which we then denote by $\wedge$. The action of Lie $\mathcal{A}_{x}$ on the module $\mathcal{A}_{x}$ is given by $\star$-multiplication as before. We now see that equation (19),

$$
\mathcal{F}(f, g)=[\mathcal{D} f \star \mathcal{D} g]-\mathcal{D}([f \stackrel{\star}{*} g]),
$$

can be written

$$
\mathcal{F} \equiv \mathbf{d}_{\star} \mathcal{A}+\mathcal{A} \wedge \mathcal{A} .
$$

The remaining equations also do not change in form (as compared to the Hochschild case): (42) implies

$$
\mathrm{d}_{\star} \mathcal{F}+\mathcal{A} \wedge \mathcal{F}-\mathcal{F} \wedge \mathcal{A}=0
$$


and the behavior under (infinitesimal )gauge transformations is

$$
\begin{aligned}
\delta \mathcal{A} & =i\left(-\mathbf{d}_{\star} \lambda+\lambda \wedge \mathcal{A}-\mathcal{A} \wedge \lambda\right) \\
\delta \mathcal{F} & =i(\lambda \wedge \mathcal{F}-\mathcal{F} \wedge \lambda) .
\end{aligned}
$$

Equations (42), (43), (44) and (45) are reminiscent of the corresponding equations of ordinary (nonabelian) gauge theory. The correspondence is given by the following dictionary: One-forms become linear operators on $\mathcal{A}_{x}$ which take one function as argument and yield a new function, two-forms become bilinear operators on $\mathcal{A}_{x}$ which take two functions as arguments and return one new function, and the Lie bracket is replaced by the antisymmetrized cup product $\wedge$. As in ordinary gauge theory, it may not be possible to use one globally defined gauge potential $\mathcal{A}$; we may need to introduce several $\mathcal{D}$ and corresponding gauge potentials $\mathcal{A}$ for functions defined on different "patches". We shall come back to this later.

Remark: One reason for going through the slightly more general Hochschild construction first is that the symmetric part of $\mathcal{F}_{H}$ may also contain interesting information as we will see in section 3.5. For invertible $\mathcal{D}$ there is still another interesting object:

$$
\widetilde{\mathcal{F}} \equiv \mathcal{D}^{-1} \circ \mathcal{F}
$$

measures noncommutativity:

$$
\widetilde{\mathcal{F}}(f, g)=\left[f^{\star^{\prime}}, g\right]-\left[f{ }^{\star}, g\right],
$$

where the (associative) product $\star^{\prime}$ is defined by $f \star^{\prime} g=\mathcal{D}^{-1}(\mathcal{D} f \star \mathcal{D} g)$. This "field strength" satisfies the Cartan-Maurer equation

$$
\mathbf{d}_{\star} \widetilde{\mathcal{F}}=[\widetilde{\mathcal{F}}, \widetilde{\mathcal{F}}]_{\mathrm{G}} .
$$

\subsubsection{Projective modules}

We shall now discuss how our formulae fit into the framework of finite projective modules: The calculus of $p$-cochains in $C^{p}$ with the coboundary operator $\mathbf{d}_{\star}$ uses only the algebraic structure of $\mathcal{A}_{x}$; it is related to the standard universal calculus and one can obtain other calculi by projection. Consider a (finite) projective right $\mathcal{A}_{x}$-module $\mathcal{E}$. We introduce a connection on $\mathcal{E}$ as a linear map $\nabla: \mathcal{E} \otimes_{\mathcal{A}_{x}} C^{p} \rightarrow \mathcal{E} \otimes_{\mathcal{A}_{x}} C^{p+1}$ for $p \in \mathbb{N}_{0}$ which satisfies the Leibniz rule

$$
\nabla(\eta \psi)=(\tilde{\nabla} \eta) \psi+(-)^{p} \eta \tilde{\mathbf{d}}_{\star} \psi
$$

for all $\eta \in \mathcal{E} \otimes_{\mathcal{A}_{x}} C^{p}, \psi \in C^{r}$, and where $\tilde{\nabla} \eta=\nabla \eta-(-)^{p} \eta \tilde{\mathbf{d}}_{\star} 1$,

$$
\tilde{\mathbf{d}}_{\star}(a \wedge \psi)=\left(\mathbf{d}_{\star} a\right) \wedge \psi+(-)^{q} a \wedge\left(\tilde{\mathbf{d}}_{\star} \psi\right)
$$

\footnotetext{
${ }^{8}$ More educated: $n$-forms become $n$-cochains. An even closer match with the usual physics conventions is achieved by multiplying our $\mathbf{d}_{\star}$ and $\mathcal{F}$ by $i$ (section 3 and later: multiply by $i \hbar$ ).
} 
for all $a \in C^{q}$, and $\tilde{\mathbf{d}}_{\star} 1$ is the identity operator on $\mathcal{A}_{x}$. (The transformation of matter fields $\hat{\delta} \psi=i \hat{\lambda} \star \psi$ leads to a slight complication here; for fields that transform in the adjoint (by star-commutator) we would only need $\tilde{\nabla}, \mathbf{d}_{\star}$ and not $\nabla$ and $\tilde{\mathbf{d}}_{\star}$.)

Let $\left(\eta_{a}\right)$ be a generating family for $\mathcal{E}$; any $\xi \in \mathcal{E}$ can then be written as $\xi=\sum \eta_{a} \psi^{a}$ with $\psi^{a} \in \mathcal{A}_{x}$ (with only a finite number of terms different from zero). For a free module the $\psi^{a}$ are unique, but we shall not assume that. Let the generalized gauge potential be defined by the action of $\tilde{\nabla}$ on the elements of the generating family: $\tilde{\nabla} \eta_{a}=\eta_{b} \mathcal{A}_{a}^{b}$. In the following we shall suppress indices and simply write $\xi=\eta . \psi, \tilde{\nabla} \eta=\eta \cdot \mathcal{A}$ etc. We compute

$$
\nabla \xi=\nabla(\eta . \psi)=\eta \cdot\left(\mathcal{A} \wedge \psi+\tilde{\mathbf{d}}_{\star} \psi\right)=\eta \cdot(\mathcal{D} \wedge \psi) .
$$

Evaluated on a function $f \in \mathcal{A}_{x}$ the component $\mathcal{D} \wedge \psi$ yields a covariant function times the matter field, $(\mathcal{D} \wedge \psi)(f)=(\mathcal{D} f) \star \psi$, so in this framework covariant functions are related to the covariant "derivative" $\tilde{\mathbf{d}}_{\star}+\mathcal{A}$ :

$$
\left[\left(\tilde{\mathbf{d}}_{\star}+\mathcal{A}\right) \psi\right](f)=(f+\mathcal{A}(f)) \star \psi .
$$

The square of the connection gives

$$
\nabla^{2} \xi=\eta \cdot\left(\mathcal{A} \wedge \mathcal{A}+\mathbf{d}_{\star} \mathcal{A}\right) \cdot \psi=\eta \cdot \mathcal{F} . \psi
$$

with the field strength

$$
\mathcal{F}=\mathbf{d}_{\star} \mathcal{A}+\mathcal{A} \wedge \mathcal{A} .
$$

\section{Ordinary versus noncommutative gauge theory}

We are particularly interested in the case where the algebra of our noncommutative space $\mathcal{A}_{x}$ is given by a star product (via a quantization map). A star product on a smooth $C^{\infty}$-Manifold $\mathcal{M}$ is an associative $\mathbb{C}[[\hbar]]$-bilinear product

$$
f \star g=f g+\sum_{n=1}^{\infty}\left(\frac{i \hbar}{2}\right)^{n} B_{n}(f, g), \quad f, g \in C^{\infty}(\mathcal{M}),
$$

where $B_{n}$ are bilinear operators and $\hbar$ is the formal deformation parameter; it is a deformation quantization of the Poisson structure

$$
\{f, g\} \equiv \theta^{i j}(x) \partial_{i} f \partial_{j} g=B_{1}(f, g)-B_{1}(g, f) .
$$

Equivalent star products $\tilde{\star}$ can be constructed with the help of invertible operators $D$

$$
D(f \tilde{\star} g)=D f \star D g, \quad D f \equiv f+\sum_{n=1}^{\infty} \hbar^{n} D_{n}(f),
$$


where $D_{n}$ are linear operators. This operation clearly does not spoil associativity. There are also inner automorphisms for each invertible element $\Lambda$ and their infinitesimal version, inner derivations,

$$
f \mapsto \Lambda \star f \star \Lambda^{-1}, \quad \delta f=[i \lambda \star f] ;
$$

these operations do not change the star product.

The striking similarity between equations (16), (17) and equations (57), (58) suggests the following interpretation of noncommutative gauge theory in the star product formalism: The covariance maps $\mathcal{D}=\mathrm{id}+\mathcal{A}$ are gauge equivalence maps $D$ for the underlying star product, combined with a change of coordinates $\rho^{*}$

$$
\mathcal{D}=D \circ \rho^{*}, \quad \mathcal{D}\left(f \star^{\prime} g\right)=\mathcal{D} f \star \mathcal{D} g
$$

gauge transformations are inner automorphisms of the star products.

\subsection{Motivation from string theory}

A Poisson tensor $\theta$ enters the discussion of Seiberg and Witten [9] via a background $B$-field in the open string picture. In this setting

$$
\theta^{i j}=2 \pi \alpha^{\prime}\left(\frac{1}{g+2 \pi \alpha^{\prime} B}\right)_{A}^{i j}
$$

appears in the propagator at boundary points of the string world sheet. ( $g$ is the closed string metric and $A$ denotes the antisymmetric part of a matrix.) The 2-form $\omega \equiv \frac{1}{2} B_{i j} d x^{i} \wedge d x^{j}$ is a symplectic form, provided $B$ is nondegenerate and $d \omega=0$, which is e.g. obviously the case if $B$ is constant (but we shall not require it to be constant.) In the zero slope limit (or in the intermediate picture with $\Phi=-B$ [9], see section 3.4)

$$
\theta^{i j}=\left(B^{-1}\right)^{i j}
$$

defines then a Poisson structure. It has been discussed by several authors how the Moyal-Weyl star product enters the picture as a quantization of this Poisson structure in the constant case [3, 4, 9]. A direct approach that is most suitable for our purposes and that also works for non-constant $\theta$ is given by Cattaneo and Felder [19 in their QFT realization of Kontsevich's star product.

In section 1.1 we have discussed what happens if we add fluctuations $f$ (with $d f=0$, i.e. locally $f=d a$ ) to the background $B$ field: The action is then naively invariant under ordinary gauge transformations $\delta a=d \lambda$, but the invariance of the quantum theory depends on the choice of regularization. A point splitting prescription [9, 10] leads in fact to a noncommutative gauge invariance. Since in a consistent quantum theory the choice of regularization should not matter, Seiberg and Witten argued that 
there should exist maps that relate ordinary and noncommutative gauge theory such that (1) holds. A more abstract argument that leads to the same conclusion, but gives the Seiberg-Witten maps more directly and also works for non-constant $\theta$ can be based on a quantum version of Moser's lemma [11, 12]. Here is briefly the idea, in the next section we will review the details: The addition of $f=d a$ to $B$ defines a new Poisson structure

$$
\theta=\frac{1}{B} \quad \rightarrow \quad \theta^{\prime}=\frac{1}{B+f}
$$

which, according to Moser's lemma, is related to the original one by a change of coordinates given by a flow $\rho_{a}^{*}$ that depends on the gauge potential $a$. After quantization $\theta$ and $\theta^{\prime}$ give rise to equivalent star products $\star$ and $\star^{\prime}$. The equivalence map $D_{a}$, the full quantum flow $\mathcal{D}_{a}=D_{a} \circ \rho_{a}^{*}$ and the noncommutative gauge potential

$$
\mathcal{A}_{a}=\mathcal{D}_{a}-\mathrm{id}
$$

are also functions of $a$. An additional infinitesimal gauge transformation

$$
\delta a=d \lambda
$$

does not change the Poisson structure (since $\delta f=0$ ), but it still induces an infinitesimal canonical transformation. After quantization that transformation becomes an inner derivation of the star product $\star$ and thus a noncommutative gauge transformation $\delta_{\hat{\lambda}}$ with $\hat{\lambda}=\hat{\lambda}(\lambda, a)$, such that

$$
\mathcal{A}_{a+d \lambda}=\mathcal{A}_{a}+\delta_{\hat{\lambda}} \mathcal{A}_{a}
$$

\subsection{Quantum version of Moser's lemma: Seiberg-Witten map}

Consider an abelian gauge theory on a manifold that also carries a Poisson structure $\theta$. The gauge potential, field strength and infinitesimal gauge transformations are

$$
a=a_{i} d x^{i}, \quad f=\frac{1}{2} f_{i j} d x^{i} \wedge d x^{j}=d a, \quad f_{i j}=\partial_{i} a_{j}-\partial_{j} a_{i}, \quad \delta_{\lambda} a=d \lambda .
$$

We will first construct a semiclassical version of the Seiberg-Witten map, where all star commutators are replaced by Poisson brackets. The construction is essentially a formal generalization of Moser's lemma to Poisson manifolds. 


\subsubsection{Semi-classical construction}

Let us consider the nilpotent coboundary operator of the Poisson cohomology (see [47]) - the semiclassical limit of (28) -

$$
\mathbf{d}_{\theta}=-[\cdot, \theta]_{\mathrm{S}},
$$

where $[,]_{\mathrm{S}}$ is the Schouten-Nijenhuis bracket (A.1) and $\theta=\frac{1}{2} \theta^{i j} \partial_{i} \wedge \partial_{j}$ is the Poisson bivector. Acting with $\mathbf{d}_{\theta}$ on a function $f$ gives the Hamiltonian vector field corresponding to $f$

$$
\mathbf{d}_{\theta} f=\{\cdot, f\}=\theta^{i j}\left(\partial_{j} f\right) \partial_{i} .
$$

It is natural to introduce a vector field

$$
\mathbf{a}_{\theta}=a_{i} \mathbf{d}_{\theta} x^{i}=\theta^{j i} a_{i} \partial_{j}
$$

corresponding to the abelian gauge potential $a$ and a bivector field

$$
\mathbf{f}_{\theta}=\mathbf{d}_{\theta} \mathbf{a}_{\theta}=-\frac{1}{2} \theta^{i k} f_{k l} \theta^{l j} \partial_{i} \wedge \partial_{j}
$$

corresponding to the abelian field strength $f=d a$. We have $\mathbf{d}_{\theta} \mathbf{f}_{\theta}=0$, due to $\mathbf{d}_{\theta}^{2} \propto$ $[\theta, \theta]_{\mathrm{S}}=0$ (Jacobi identity).

We are now ready to perturb the Poisson structure $\theta$ by introducing a one-parameter deformation $\theta_{t}$ with $t \in[0,1]:$ :

$$
\partial_{t} \theta_{t}=\mathbf{f}_{\theta_{t}}
$$

with initial condition $\theta_{0}=\theta$. In local coordinates:

$$
\partial_{t} \theta_{t}^{i j}=-\left(\theta_{t} f \theta_{t}\right)^{i j}, \quad \theta_{0}^{i j}=\theta^{i j}
$$

with formal solution given by the geometric series

$$
\theta_{t}=\theta-t \theta f \theta+t^{2} \theta f \theta f \theta-t^{3} \theta f \theta f \theta f \theta \pm \cdots=\theta \frac{1}{1+t f \theta}
$$

if $f$ is not explicitly $\theta$-dependent. (The differential equations (71), (72) and the rest of the construction do make sense even if $f$ or $a$ are $\theta$-dependent). $\theta_{t}$ is a Poisson tensor for all $t$ because $\left[\theta_{t}, \theta_{t}\right]_{\mathrm{S}}=0$ at $t=0$ and

$$
\partial_{t}\left[\theta_{t}, \theta_{t}\right]_{\mathrm{S}}=-2 \mathbf{d}_{\theta_{t}} \mathbf{f}_{\theta_{t}} \propto\left[\theta_{t}, \theta_{t}\right]_{\mathrm{S}}
$$

\footnotetext{
${ }^{9}$ In this notation the equations resemble those of Moser's original lemma, which deals with the symplectic 2 -form $\omega$, the inverse of $\theta$ (provided it exists). There, e.g., $\partial_{t} \omega_{t}=f$ for $\omega_{t}=\omega+t f$.
} 
The evolution (71) of $\theta_{t}$ is generated by the vector field $\mathbf{a}_{\theta}$ :

$$
\partial_{t} \theta_{t}=\mathbf{d}_{\theta_{t}} \mathbf{a}_{\theta_{t}}=-\left[\mathbf{a}_{\theta_{t}}, \theta_{t}\right]_{\mathrm{S}} .
$$

This Lie derivative can be integrated to a flow (see appendix A.3)

$$
\rho_{a}^{*}=\left.\exp \left(\mathbf{a}_{\theta_{t}}+\partial_{t}\right) \exp \left(-\partial_{t}\right)\right|_{t=0}
$$

that relates the Poisson structures $\theta^{\prime}=\theta_{1}$ and $\theta=\theta_{0}$. In analogy to (32) we define a semi-classical (semi-noncommutative) generalized gauge potential

$$
A_{a}=\rho_{a}^{*}-\mathrm{id} .
$$

Under an infinitesimal gauge transformation $a \mapsto a+d \lambda$ the vector field (69) changes by a Hamiltonian vector field $\mathbf{d}_{\theta} \lambda=\theta^{i j}\left(\partial_{j} \lambda\right) \partial_{i}$ :

$$
\mathbf{a}_{\theta} \mapsto \mathbf{a}_{\theta}+\mathbf{d}_{\theta} \lambda .
$$

Let us compute the effect of this gauge transformation on the flow (75)). After some computation (see appendix A.4) we find (infinitesimally: to first order in $\lambda$ )

$$
\rho_{a+d \lambda}^{*}=\left(\mathrm{id}+\mathbf{d}_{\theta} \tilde{\lambda}\right) \circ \rho_{a}^{*}, \quad \text { i.e., } \quad \rho_{a+d \lambda}^{*}(f)=\rho_{a}^{*}(f)+\left\{\rho_{a}^{*}(f), \tilde{\lambda}\right\}
$$

and

$$
A_{a+d \lambda}=A_{a}+\mathbf{d}_{\theta} \tilde{\lambda}+\left\{A_{a}, \tilde{\lambda}\right\},
$$

with

$$
\tilde{\lambda}(\lambda, a)=\left.\sum_{n=0}^{\infty} \frac{\left(\mathbf{a}_{\theta_{t}}+\partial_{t}\right)^{n}(\lambda)}{(n+1) !}\right|_{t=0} .
$$

Equations (80) and (76) with (75) are explicit semi-classical versions of the SeibergWitten map. The semi-classical (semi-noncommutative) generalized field strength evaluated on two functions (e.g. coordinates) $f, g$ is

$$
F_{a}(f, g)=\left\{\rho^{*} f, \rho^{*} g\right\}-\rho^{*}\{f, g\}=\rho^{*}\left(\{f, g\}^{\prime}-\{f, g\}\right) .
$$

Abstractly as 2-cochain:

$$
F_{a}=\rho^{*} \circ \frac{1}{2}\left(\theta^{\prime}-\theta\right)^{i k} \partial_{i} \wedge \partial_{k}=\rho^{*} \circ \frac{1}{2}\left(f^{\prime}\right)_{j l} \theta^{i j} \theta^{k l} \partial_{i} \wedge \partial_{k}
$$

with $\theta^{\prime} f=\theta f^{\prime}$, or

$$
f^{\prime}=\frac{1}{1+f \theta} f
$$


which we recognize as the noncommutative field strength (with lower indices) for constant $f, \theta$ [9]. The general result for non-constant $f, \theta$ is thus simply obtained by the application of the covariantizing map $\rho^{*}$ (after raising indices with $\theta$ 's).

The Seiberg-Witten map in the semiclassical regime for constant $\theta$ has previously been discussed in [33, 35], where it was understood as a coordinate redefinition that eliminates fluctuations around a constant background.

We will now use Kontsevich's formality theorem to quantize everything. The goal is to obtain (畐) in the form (65) of which (79) is the semi-classical limit.

\subsubsection{Kontsevich formality map}

Kontsevich's formality map is a collection of skew-symmetric multilinear maps $U_{n}$ for $n=0 \ldots \infty$ that map tensor products of $n$ polyvector fields to differential operators. More precisely $U_{n}$ maps the tensor product of $n k_{i}$-vector fields to an $m$-differential operator, where $m$ is determined by the matching condition

$$
m=2-2 n+\sum_{i=1}^{n} k_{i}
$$

$U_{1}$ in particular is the natural map from a $k$-vector field to a $k$-differential operator

$$
U_{1}\left(\xi_{1} \wedge \ldots \wedge \xi_{k}\right)\left(f_{1}, \ldots, f_{k}\right)=\frac{1}{k !} \sum_{\sigma \in \Sigma_{k}} \operatorname{sgn}(\sigma) \prod_{i=1}^{k} \xi_{\sigma_{i}}\left(f_{i}\right),
$$

and $U_{0}$ is defined to be the ordinary multiplication of functions:

$$
U_{0}(f, g)=f g .
$$

The $U_{n}, n \geq 1$, satisfy the formality condition [13]

$$
\begin{array}{r}
d_{\mu} U_{n}\left(\alpha_{1}, \ldots, \alpha_{n}\right)+\frac{1}{2} \sum_{\substack{I \sqcup J=(1, \ldots, n) \\
I, J \neq \emptyset}} \pm\left[U_{|I|}\left(\alpha_{I}\right), U_{|J|}\left(\alpha_{J}\right)\right]_{\mathrm{G}} \\
=\sum_{i<j} \pm U_{n-1}\left(\left[\alpha_{i}, \alpha_{j}\right]_{\mathrm{S}}, \alpha_{1}, \ldots, \widehat{\alpha}_{i}, \ldots, \widehat{\alpha}_{j}, \ldots, \alpha_{n}\right),
\end{array}
$$

where $d_{\mu} \mathcal{C} \equiv-[\mathcal{C}, \mu]_{\mathrm{G}}$, with the commutative multiplication $\mu(f, g)=f \cdot g$ of functions; the hat marks an omitted vector field. See [13, 19] for explicit constructions and more details and [37, 13] for the definition of the signs in this equation. In the following we collect the three special cases that we actually use in this paper.

Consider the formal series (see also [38)

$$
\Phi(\alpha)=\sum_{n=0}^{\infty} \frac{(i \hbar)^{n}}{n !} U_{n+1}(\alpha, \theta, \ldots, \theta) .
$$


According to the matching condition (84), $U_{n+1}(\alpha, \theta, \ldots, \theta)$ is a tridifferential operator for every $n$ if $\alpha$ is a trivector field, it is a bidifferential operator if $\alpha$ is a bivector field, it is a differential operator if $\alpha$ is a vector field and it is a function if $\alpha$ is a function; in all cases $\theta$ is assumed to be a bidifferential operator.

Star products from Poisson tensors A Poisson bivector $\theta$ gives rise to a star product via the formality map: According to the matching condition (84), $U_{n}(\theta, \ldots, \theta)$ is a bidifferential operator for every $n$ if $\theta$ is a bivector field. This can be used to define a product

$$
f \star g=\sum_{n=0}^{\infty} \frac{(i \hbar)^{n}}{n !} U_{n}(\theta, \ldots, \theta)(f, g)=f g+\frac{i \hbar}{2} \theta^{i j} \partial_{i} f \partial_{j} g+\cdots
$$

The formality condition implies

$$
\mathbf{d}_{\star} \star=i \hbar \Phi\left(\mathbf{d}_{\theta} \theta\right)
$$

or, $[\star, \star]_{\mathrm{G}}=i \hbar \Phi\left([\theta, \theta]_{\mathrm{S}}\right)$, i.e., associativity of $\star$, if $\theta$ is Poisson. (If $\theta$ is not Poisson, i.e., has non-vanishing Schouten-Nijenhuis bracket $[\theta, \theta]_{\mathrm{S}}$, then the product $\star$ is not associative, but the non-associativity is nevertheless under control via the formality condition by (90).)

Differential operators from vector fields We can define a linear differential operator $^{10}$

$$
\Phi(\xi)=\xi+\frac{(i \hbar)^{2}}{2} U_{3}(\xi, \theta, \theta)+\cdots
$$

for every vector field $\xi$. For $\theta$ Poisson the formality condition gives

$$
\mathbf{d}_{\star} \Phi(\xi)=i \hbar \Phi\left(\mathbf{d}_{\theta} \xi\right)=i \hbar \mathbf{d}_{\theta} \xi+\cdots .
$$

Vector fields $\xi$ that preserve the Poisson bracket, $\mathbf{d}_{\theta} \xi=-[\theta, \xi]_{\mathrm{S}}=0$, give rise to derivations of the star product (89): From (92) and the definition (A.7), (29) of $\mathbf{d}_{\star}$

$$
0=\left[\mathbf{d}_{\star} \Phi(\xi)\right](f, g)=-[\Phi(\xi)](f \star g)+f \star[\Phi(\xi)](g)+[\Phi(\xi)](f) \star g .
$$

Inner derivations from Hamiltonian vector fields Hamiltonian vector fields $\mathbf{d}_{\theta} f$ give rise to inner derivations of the star product (89): We can define a new function $\square$

$$
\hat{f} \equiv \Phi(f)=f+\frac{(i \hbar)^{2}}{2} U_{3}(f, \theta, \theta)+\cdots
$$

\footnotetext{
${ }^{10} U_{2}(\xi, \theta)=0$ and $U_{2}(f, \theta)=0$ by explicit computation of Kontsevich's formulas.
} 
for every function $f$. For $\theta$ Poisson the formality condition gives

$$
\mathbf{d}_{\star} \hat{f}=i \hbar \Phi\left(\mathbf{d}_{\theta} f\right)
$$

Evaluated on a function $g$, this reads

$$
\left[\Phi\left(\mathbf{d}_{\theta} f\right)\right](g)=\frac{1}{i \hbar}[g \stackrel{\star}{,} \hat{f}] .
$$

The Hamiltonian vector field $\mathbf{d}_{\theta} f$ is thus mapped to the inner derivation $\frac{i}{\hbar}\left[\hat{f}^{\star}, \cdot\right]$.

\subsubsection{Quantum construction}

The construction mirrors the semiclassical one, the exact correspondence is given by the formality maps $U_{n}$ that are skew-symmetric multilinear maps that take $n$ polyvector fields into a polydifferential operator. We start with the differential operator

$$
\mathbf{a}_{\star}=\sum_{n=0}^{\infty} \frac{(i \hbar)^{n}}{n !} U_{n+1}\left(\mathbf{a}_{\theta}, \theta, \ldots, \theta\right),
$$

which is the image of the vector field $\mathbf{a}_{\theta}$ under the formality map (91); then we use the coboundary operator $\mathbf{d}_{\star}(28)$ to define a bidifferential operator

$$
f_{\star}=d_{\star} a_{\star} .
$$

This is the image of $\mathbf{f}_{\theta}=\mathbf{d}_{\theta} \mathbf{a}_{\theta}$ under the formality map:

$$
\mathbf{f}_{\star}=\sum_{n=0}^{\infty} \frac{(i \hbar)^{n+1}}{n !} U_{n+1}\left(\mathbf{f}_{\theta}, \theta, \ldots, \theta\right) .
$$

A $t$-dependent Poisson structure (71) induces a $t$-dependent star product via (89)

$$
g \star_{t} h=\sum_{n=0}^{\infty} \frac{(i \hbar)^{n}}{n !} U_{n}\left(\theta_{t}, \ldots, \theta_{t}\right)(g, h) .
$$

The $t$-derivative of this equation is

$$
\partial_{t}\left(g \star_{t} h\right)=\sum_{n=0}^{\infty} \frac{(i \hbar)^{n+1}}{n !} U_{n+1}\left(\mathbf{f}_{\theta_{t}}, \theta_{t}, \ldots, \theta_{t}\right)(g, h),
$$

where we have used (71) and the skew-symmetry and multi-linearity of $U_{n}$. Comparing with (99) we find

$$
\partial_{t}\left(g \star_{t} h\right)=\mathbf{f}_{\star t}(g, h),
$$


or, shorter, as an operator equation: $\partial_{t}\left(\star_{t}\right)=\mathbf{f}_{\star_{t}}$. But $\mathbf{f}_{\star_{t}}=\mathbf{d}_{\star_{t}} \mathbf{a}_{\star t}=-\left[\mathbf{a}_{\star_{t}}, \star_{t}\right]_{\mathrm{G}}$, so the $t$-evolution is generated by the differential operator $\mathbf{a}_{\star_{t}}$ and can be integrated to a flow (see appendix A.3)

$$
\mathcal{D}_{a}=\left.\exp \left(\mathbf{a}_{\star_{t}}+\partial_{t}\right) \exp \left(-\partial_{t}\right)\right|_{t=0},
$$

that relates the star products $\star^{\prime}=\star_{1}$ and $\star=\star_{0}$, and that defines the generalized noncommutative gauge potential

$$
\mathcal{A}_{a}=\mathcal{D}_{a}-\mathrm{id}
$$

The transformation of $\mathbf{a}_{\star}$ under an infinitesimal gauge transformation $a \mapsto a+d \lambda$ can be computed from (77) with the help of (95), see (31):

$$
\mathbf{a}_{\star} \mapsto \mathbf{a}_{\star}+\frac{1}{i \hbar} \mathbf{d}_{\star} \hat{\lambda}
$$

The effect of this transformation on the quantum flow and on the noncommutative gauge potential are (see appendix A.4)

$$
\begin{aligned}
& \mathcal{D}_{a+d \lambda}=\left(\mathrm{id}+\frac{1}{i \hbar} \mathbf{d}_{\star} \hat{\Lambda}\right) \circ \mathcal{D}_{a}, \quad \text { i.e., } \quad \mathcal{D}_{a+d \lambda}(f)=\mathcal{D}_{a} f+\frac{i}{\hbar}\left[\hat{\Lambda} \star \mathcal{D}_{a} f\right] \\
& \mathcal{A}_{a+d \lambda}=\mathcal{A}_{a}+\frac{1}{i \hbar}\left(\mathbf{d}_{\star} \hat{\Lambda}-\hat{\Lambda} \star \mathcal{A}+\mathcal{A} \star \hat{\Lambda}\right) .
\end{aligned}
$$

with

$$
\hat{\Lambda}(\lambda, a)=\left.\sum_{n=0}^{\infty} \frac{\left(\mathbf{a}_{\star t}+\partial_{t}\right)^{n}(\hat{\lambda})}{(n+1) !}\right|_{t=0} .
$$

Equations (104) with (103) and (108) are explicit versions of the abelian Seiberg-Witten map to all orders in $\hbar$. They are unique up to (noncommutative) gauge transformations. Perhaps more importantly this construction provides us with an explicit version of the "covariantizer" $\mathcal{D}_{a}$ (the equivalence map that sends coordinates and functions to their covariant analogs) in terms of a finite number of (classical) fields $a_{i}$. The noncommutative gauge parameter (108) also satisfies the consistency condition

$$
\delta_{\alpha} \hat{\Lambda}(\beta, a)-\delta_{\beta} \hat{\Lambda}(\alpha, a)=\frac{i}{\hbar}[\hat{\Lambda}(\alpha, a) \stackrel{\star}{,} \hat{\Lambda}(\beta, a)],
$$

with $\delta_{\alpha}\left(a_{i}\right)=\partial_{i} \alpha, \delta_{\alpha}(\beta)=0$, that follows from computing the commutator of abelian gauge transformations on a covariant field [16].

The generalized noncommutative field strength evaluated on two functions (or coordinates) $f, g$ is

$$
\mathcal{F}_{a}(f, g)=\mathcal{D}_{a}\left(\left[f \star^{\prime}, g\right]-[f \stackrel{\star}{,} g]\right) .
$$


Up to order $\theta^{2}$ the series for $\mathcal{A}_{a}$ and $\Lambda$ agree with the semiclassical results. In components:

$$
\begin{aligned}
& \mathcal{A}_{a}\left(x^{i}\right)=\theta^{i j} a_{j}+\frac{1}{2} \theta^{k l} a_{l}\left(\partial_{k}\left(\theta^{i j} a_{j}\right)-\theta^{i j} f_{j k}\right)+\ldots \\
& \hat{\Lambda}=\lambda+\frac{1}{2} \theta^{i j} a_{j} \partial_{i} \lambda+\frac{1}{6} \theta^{k l} a_{l}\left(\partial_{k}\left(\theta^{i j} a_{j} \partial_{i} \lambda\right)-\theta^{i j} f_{j k} \partial_{i} \lambda\right)+\ldots
\end{aligned}
$$

There are three major strategies for the computation of the Seiberg-Witten map:

(i) From the gauge equivalence condition (11) one can directly obtain recursion relations for the terms in the Seiberg-Witten map. For constant $\theta$ these can be cast in the form of differential equations [9]. Terms of low order in the gauge fields but all orders in $\theta$ can be expressed in terms of $\star_{n}$-products 41, 42. 1.

(ii) A path integral approach can be based on the relationship between open Wilson lines in the commutative and noncommutative picture [31, 44].

(iii) The equivalence of the star products corresponding to the perturbed and unperturbed Poisson structures leads to our formulation in the framework of deformation quantization. This allows a closed formula for the Seiberg-Witten map to all orders in the gauge fields and in $\theta$.

\subsection{Covariance and (non) uniqueness}

The objects $\mathcal{D}_{a}$ and $\hat{\Lambda}$ are not unique if all we ask is that they satisfy the generalized Seiberg-Witten condition (106) with star product $\star$ and have the correct "classical limit" $\mathcal{D}=\mathbf{a}_{\theta}+\ldots, \hat{\Lambda}=\lambda+\ldots$ The pair $\mathcal{D}_{2} \circ \mathcal{D}_{a} \circ \mathcal{D}_{1}, \mathcal{D}_{2}(\hat{\Lambda})$, where $\mathcal{D}_{2}$ is an *-algebra automorphisms and $\mathcal{D}_{1}$ is an equivalence map (possibly combined with a change of coordinates of the form id $\left.+o\left(\theta^{2}\right)\right)$ is an equally valid solution. If we allow also a transformation to a new (but equivalent) star product $₹$ then we can relax the condition on $\mathcal{D}_{2}$ : it may be any fixed equivalence map possibly combined with a change of coordinates. The maps (differential operators) $\mathcal{D}_{1}$ and $\mathcal{D}_{2}$ may depend on the gauge potential $a_{i}$ via $f_{i j}$; it is important, however, that they are gauge-invariant. The freedom in the choice of $\mathcal{D}_{1}$ and $\mathcal{D}_{2}$ represents the freedom in the choice of coordinates and/or quantization scheme in our construction; different $\mathcal{D}_{a}, \hat{\Lambda}$ related by $\mathcal{D}_{1}, \mathcal{D}_{2}$ should be regarded as being equivalent.

Figure 1 illustrates how the semi-classical and quantum constructions are affected by a change of coordinates $\sigma^{*}$ : The quantization of $\bar{\theta}$ and $\overline{\theta^{\prime}}$ in the new coordinates

\footnotetext{
${ }^{11}$ Some motivation for the latter was provided in [40, and 43] provided some more concrete understanding of the relationship of the generalized star product and Seiberg-Witten map.
} 


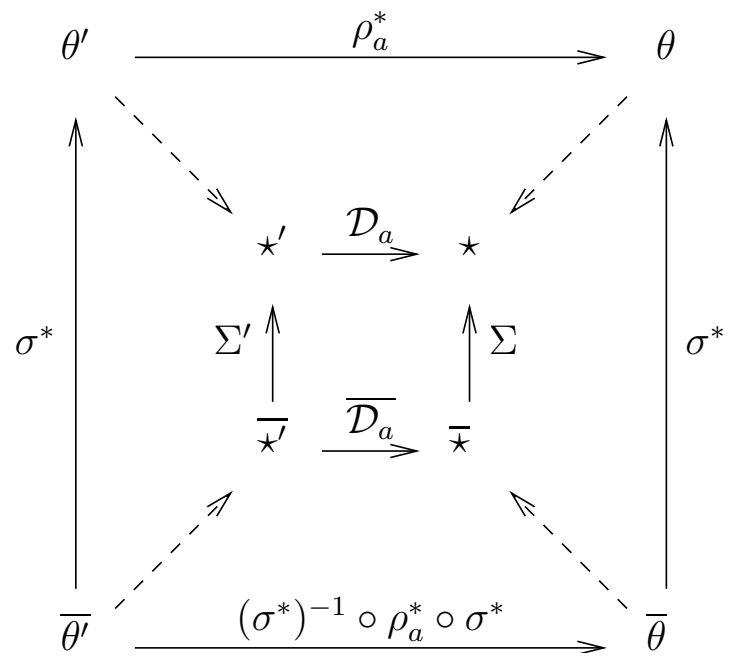

Figure 1: Two nested commutative diagrams that illustrate the covariance of the semiclassical and quantum constructions under a change of coordinates given by $\sigma^{*}$. The dashed lines indicate Kontsevich quantization. $\Sigma$ and $\Sigma^{\prime}$ are the equivalence maps (including $\sigma^{*}$ ) that relate the star products that were computed in the new coordinates with those computed in the old coordinates. The top and bottom trapezia illustrate the construction of the covariantizing equivalence maps in the old and new coordinates.

leads to star products $\bar{\star}$ and $\overline{\star^{\prime}}$ that are related to the star products $\star$ and $\star^{\prime}$ in the old coordinates by equivalence maps $\Sigma$ and $\Sigma^{\prime}$ respectively. (We have included $\sigma^{*}$ in the definition of these maps.) Note that in general $\Sigma \neq \Sigma^{\prime}$. The covariantizing equivalence map, generalized gauge potential and field strength in the new coordinates and old coordinates are related by:

$$
\begin{aligned}
& \overline{\mathcal{D}_{a}}=\Sigma^{-1} \circ \mathcal{D}_{a} \circ \Sigma^{\prime}, \\
& \overline{\mathcal{A}_{a}}=\Sigma^{-1} \circ\left(\Sigma^{\prime}-\Sigma\right)+\Sigma^{-1} \circ \mathcal{A}_{a} \circ \Sigma^{\prime}, \\
& \overline{\mathcal{F}_{a}}=\Sigma^{-1} \circ \mathcal{F}_{a} \circ\left(\Sigma^{\prime}\right)^{\otimes 2} .
\end{aligned}
$$

Explicit (but complicated) expressions for $\Sigma$ and $\Sigma^{\prime}$ in terms of $\theta, \sigma^{*}$, and the gauge potential $a$ can be computed with methods similar to the ones that we have used to compute $\mathcal{D}_{a}$ in the previous section.

\subsection{Dirac-Born-Infeld action in the intermediate picture}

Seiberg and Witten have argued that the open string theory effective action in the presence of a background $B$-field can be expressed either in terms of ordinary gauge theory written in terms of the combination $B+F$ or in terms of noncommutative gauge 
theory with gauge field $\widehat{F}$, where the $B$-dependence appears only via the $\theta$-dependence of the star product and the open string metric $G$ and effective coupling $G_{s}$. (Here we implicitly need to assume that $\theta$ is Poisson, which is of course the case for constant $\theta$.) There is also an intermediate picture with an effective noncommutative action which is a function of $\widehat{\Phi}+\widehat{F}$, where $\widehat{\Phi}$ is a covariant version of some antisymmetric matrix $\Phi$, with a $\theta$-dependent star product and effective metric $G$ and string coupling $G_{s}$. The proposed relations between the new quantities and the background field $B$, the given closed string metric $g$ and the coupling $g_{s}$ are

$$
\frac{1}{G+\Phi}=\frac{1}{g+B}-\theta, \quad \frac{\operatorname{det}^{\frac{1}{2}}(g+B)}{g_{s}}=\frac{\operatorname{det}^{\frac{1}{2}}(G+\Phi)}{G_{s}} .
$$

The first relation can also be written more symmetrically:

$$
[1+(G+\Phi) \theta][1-(g+B) \theta]=1 ; \quad G_{s}=g_{s} \operatorname{det}^{-\frac{1}{2}}[1-(g+B) \theta] .
$$

Given $g$ and $B$ we can pick essentially any antisymmetric matrix $\theta$ - in particular one that satisfies the Jacobi identity - and find $G$ and $\Phi$ as symmetric and antisymmetric parts of the following expression

$$
G+\Phi=\frac{1}{1-(g+B) \theta}(g+B)
$$

For $\theta=1 / B$ (as in the zero slope limit): $G=-B g^{-1} B, \Phi=-B, G_{s}=g_{s} \operatorname{det}^{\frac{1}{2}}\left(-B g^{-1}\right)$.

For slowly varying but not necessarily small fields on a $D$-brane the effective theory is given by the Dirac-Born-Infeld (DBI) action. In the following we will show that the ordinary DBI action is exactly equal to the semi-noncommutative DBI action in the intermediate picture. There are no derivative corrections. By semi-noncommutative we mean the semiclassical limit of a noncommutative theory with star commutators, e.g. in the noncommutative transformation law, replaced by Poisson brackets as in section 3.2.1. Using (117) we can derive the following identity for scalar densities

$$
\frac{1}{g_{s}} \operatorname{det}^{\frac{1}{2}}(g+B+F)=\frac{1}{G_{s}} \operatorname{det}^{\frac{1}{2}}\left(\frac{\theta}{\theta^{\prime}}\right) \operatorname{det}^{\frac{1}{2}}\left(G+\Phi+F^{\prime}\right)
$$

where

$$
\theta^{\prime}=\theta \frac{1}{1+F \theta}, \quad F^{\prime}=\frac{1}{1+F \theta} F
$$

\footnotetext{
${ }^{12}$ To avoid confusion with the matrices we will use bold face letters for tensors and forms in this section (e.g.: $\boldsymbol{\theta}=\frac{1}{2} \theta^{i j} \partial_{i} \wedge \partial_{j}, \boldsymbol{\omega}=\frac{1}{2} \omega_{i j} d x^{i} \wedge d x^{j}, \theta=\omega^{-1}$ ); for simplicity we shall assume that all matrices are nondegenerate when needed.
} 
(understood as formal power series). Raising indices on $F^{\prime}$ with $\theta$ we get $\theta^{\prime}-\theta \equiv$ $\left\{x^{i}, x^{j}\right\}^{\prime}-\left\{x^{i}, x^{j}\right\}$ which we recognize as the semiclassical version of

$$
\tilde{F}^{i j}=\left[x^{i}, \star^{\prime}, x^{j}\right]-\left[x^{i}, x^{j}\right],
$$

compare equation (47). The semi-noncommutative field strength changes by canonical transformation under gauge transformations (78); it is obtained from the invariant $\theta^{\prime}-\theta$ by action of the covariantizing $\rho^{*}$ (see also (19), (81)):

$$
\rho^{*}\left(\theta^{\prime}-\theta\right)=\left\{\rho^{*} x^{i}, \rho^{*} x^{j}\right\}-\rho^{*}\left(\left\{x^{i}, x^{j}\right\}\right) .
$$

The corresponding object with lower indices is

$$
\widehat{F}=\rho^{*}\left(F^{\prime}\right) \text {. }
$$

The Poisson structures $\boldsymbol{\theta}^{\prime}$ and $\boldsymbol{\theta}$ are related by the change of coordinates $\rho^{*}: \rho^{*} \boldsymbol{\theta}^{\prime}=\boldsymbol{\theta}$. The matrices $\theta^{\prime}, \theta$ are consequently related to the Jacobian $\operatorname{det}\left(\partial \rho^{*}(x) / \partial x\right)$ of $\rho^{*}$ :

$$
\operatorname{det}^{\frac{1}{2}}\left(\frac{\theta}{\rho^{*} \theta^{\prime}}\right) \cdot \operatorname{det}\left(\frac{\partial \rho^{*}(x)}{\partial x}\right)=1 \text {. }
$$

Using this we can derive the following exact equality for the DBI action with background field $B$ and the semi-noncommutative DBI action without $B$ (but with $\Phi$ ) in the intermediate picture,

$$
\int d^{p} x \frac{1}{g_{s}} \operatorname{det}^{\frac{1}{2}}(g+B+F)=\int d^{p} x \frac{1}{\widehat{G}_{s}} \frac{\operatorname{det}^{\frac{1}{2}}\left(\rho^{*} \theta\right)}{\operatorname{det}^{\frac{1}{2}} \theta} \operatorname{det}^{\frac{1}{2}}(\widehat{G}+\widehat{\Phi}+\widehat{F}),
$$

with covariant $\widehat{G}_{s} \equiv \rho^{*} G_{s}, \widehat{G} \equiv \rho^{*} G, \widehat{\Phi} \equiv \rho^{*} \Phi, \widehat{F}=\rho^{*} F^{\prime}$ that transform semi-classically under gauge transformations (78). The only object without a " $\rho^{*}$ ", $\operatorname{det}^{-\frac{1}{2}} \theta$, is important since it ensures that the semi-noncommutative action is invariant under gauge transformations, i.e. canonical transformations. The factor $\operatorname{det}^{\frac{1}{2}}\left(\rho^{*} \theta\right) / \operatorname{det}^{\frac{1}{2}}(\theta)$ can be absorbed in a redefinition of $G_{s}$; it is equal to one in the case of constant $\theta$, since $\rho^{*}$ does not change a constant;

$$
\int d^{p} x \frac{1}{g_{s}} \operatorname{det}^{\frac{1}{2}}(g+B+F)=\int d^{p} x \frac{1}{\widehat{G}_{s}} \operatorname{det}^{\frac{1}{2}}(\widehat{G}+\widehat{\Phi}+\widehat{F}) \quad(\theta \text { const. }) .
$$

General actions invariant under the semiclassical Seiberg-Witten map, which includes the Born-Infleld action as well as some actions with derivative terms, have been discussed in [34] in the case of constant $\theta=B^{-1}$ and constant metric $g$.

One can consider a fully noncommutative version of the DBI action with $\rho^{*}$ replaced by the equivalence map $\mathcal{D}$ and with star products in the appropriate places. That action no longer exactly equals its commutative cousin but differs only by derivative terms as can be seen [39] by using the explicit form of the equivalence map (103) - 
ordering ambiguities in the definition of the action also contribute only derivative terms. The equivalence up to derivative terms of the commutative and noncommutative DBI actions was previously shown by direct computation [9] in the case of constant $\theta$; in this case an alternative derivation that is closer to our present discussion and is based on a conjectured formula for the Seiberg-Witten map was given in 42]. Requireing equivalence of the commutative and noncommutative descriptions one can compute derivative corrections to the DBI action [36].

The semi-noncommutative actions have the general form

$$
S_{\Phi}=\int d^{p} x \frac{1}{\operatorname{det}^{\frac{1}{2}} \theta} \rho_{(\theta, a)}^{*}\left(\mathcal{L}\left(G_{s}, G, \Phi, \theta^{\prime}, \theta\right)\right)
$$

where $\mathcal{L}$ is a gauge invariant scalar function. The gauge potential enters in two places: in $\theta^{\prime}$ via the gauge invariant field strength $f$ and in $\rho_{(\theta, a)}^{*}$. It is interesting to note that even the metric and the coupling constants will in general transform under gauge transformations since they depend on the gauge potential via $\rho^{*}$. Under gauge transformations $\rho^{*} \mathcal{L}$ transforms canonically:

$$
\delta_{\lambda}\left(\rho^{*} \mathcal{L}\right)=\left\{\rho^{*} \mathcal{L}, \tilde{\lambda}\right\}
$$

Due to the special scalar density $\operatorname{det}^{-\frac{1}{2}} \theta$, the action $S_{\Phi}$ is gauge invariant and covariant under general coordinate transformations.

In the "background independent" gauge $\theta=B^{-1}, \Phi=-B$ [32] the action (125) becomes simply

$$
S_{\mathrm{DBI}}=\int d^{p} x \frac{1}{\operatorname{det}^{\frac{1}{2}} \theta} \rho^{*}\left(\frac{1}{g_{s}} \operatorname{det}^{\frac{1}{2}}\left(1+g \theta^{\prime}\right)\right),
$$

with $\theta^{\prime}=(B+F)^{-1}$. Expanding the determinant to lowest nontrivial order we find the following semi-noncommutative Yang-Mills action

$$
\int d^{p} x \frac{1}{\operatorname{det}^{\frac{1}{2}} \theta} \rho^{*}\left(\frac{1}{4 g_{s}} g_{i j} \theta^{\prime j k} g_{k l} \theta^{\prime l i}\right)=\int d^{p} x \frac{1}{\operatorname{det}^{\frac{1}{2}} \theta} \frac{1}{4 \hat{g}_{s}} \hat{g}_{i j}\left\{\hat{x}^{j}, \hat{x}^{k}\right\} \hat{g}_{k l}\left\{\hat{x}^{l}, \hat{x}^{i}\right\},
$$

with covariant coupling constant $\hat{g}_{s}=\rho^{*} g_{s}$, metric $\hat{g}_{i j}=\rho^{*} g_{i j}$ and coordinates $\hat{x}^{i}=$ $\rho^{*} x^{i}$. An analogous fully noncommutative version can be written with the help of the covariantizing equivalence map $\mathcal{D}$

$$
S_{\mathrm{NC}}=\int d^{p} x \operatorname{det}^{\frac{1}{2}} \omega \mathcal{D}\left(\frac{1}{4 g_{s}} \star^{\prime} g_{i j} \star^{\prime} \tilde{F}^{j k} \star^{\prime} g_{k l} \star^{\prime} \tilde{F}^{l i}\right)
$$

with an appropriate scalar density $\operatorname{det}^{\frac{1}{2}} \omega$ that ensures upon integration a cyclic trace (this is important for the gauge invariance of the action.) In the zero-slope limit $\tilde{F}$ is 
as given in (121), in the background independent gauge $\tilde{F}^{i j}=\left[x^{i} \star^{\prime}, x^{j}\right]$. In the latter case

$$
S_{\mathrm{NC}}=\int d^{p} x \operatorname{det}^{\frac{1}{2}} \omega \frac{1}{4 \hat{g}_{s}} \star \hat{g}_{i j} \star\left[\hat{X}^{j} \star \hat{X}^{k}\right] \star \hat{g}_{k l} \star\left[\hat{X}^{l} \star \hat{X}^{i}\right] .
$$

This has the form of a matrix model potential (albeit with nonconstant $g_{s}, g$ ) with covariant coordinates $\hat{X}^{i}=\mathcal{D} x^{i}$ as dynamical variables.

\subsection{Some notes on symmetric tensors}

The matrix

$$
\tilde{\theta}^{i j}=\left(\frac{1}{B+g}\right)^{i j}=\theta^{i j}+G^{i j}
$$

plays a central role for strings in a background $B$-field (with $\Phi=0$ ): its symmetric part $\tilde{\theta}_{S}^{i j}$ is the effective open string metric and its antisymmetric part $\tilde{\theta}_{A}^{i j}$ provides the Poisson structure (provided it is indeed Poisson) that leads upon quantization to the noncommutativity felt by the open strings. One may now ask out of pure curiosity whether it is possible to quantize $\tilde{\theta}$ directly, i.e., whether it is possible to find an associative star product $\tilde{\star}$ which in lowest order in $\hbar$ is given by $\tilde{\theta}$ (which is not antisymmetric):

$$
f \tilde{\star} g=f g+\frac{i \hbar}{2} \tilde{\theta}^{i j} \partial_{i} f \partial_{j} g+o\left(\hbar^{2}\right) .
$$

This is indeed possible, provided $\tilde{\theta}_{A}$ is Poisson (i.e. satisfies the Jacobi identity), since the symmetric part of the star product can be gauged away by an equivalence map

$$
\Xi(f \tilde{\star} g)=\Xi(f) \star \Xi(g)
$$

where

$$
f \star g=f g+\frac{i \hbar}{2} \tilde{\theta}_{A}^{i j} \partial_{i} f \partial_{j} g+o\left(\hbar^{2}\right) .
$$

An equivalence map that does the job can be given explicitly in terms of the symmetric part of $\tilde{\theta}$ :

$$
\Xi=\exp \left(-\frac{i \hbar}{4} \tilde{\theta}_{S}^{i j} \partial_{i} \partial_{j}\right)
$$

It is enough to check terms up to order $\hbar$

$$
\begin{aligned}
& f g+\frac{i \hbar}{2} \tilde{\theta}^{i j} \partial_{i} f \partial_{j} g-\frac{i \hbar}{4} \tilde{\theta}_{S}^{i j} \partial_{i} \partial_{j}(f g) \\
& =f g+\frac{i \hbar}{2} \tilde{\theta}_{A}^{i j} \partial_{i} f \partial_{j} g-\frac{i \hbar}{4} \tilde{\theta}_{S}^{i j}\left[\left(\partial_{i} \partial_{j} f\right) g+f\left(\partial_{i} \partial_{j} g\right)\right] .
\end{aligned}
$$


To quantize $\tilde{\theta}$ we can thus proceed as follows: first one quantizes $\tilde{\theta}_{A}$ e.g. with Kontsevich's formula and then one uses $\Xi$ to get $\tilde{\star}$ from $\star$. In the previous sections we saw that the full information about the noncommutative gauge fields is encoded in the equivalence map $\mathcal{D}_{a}$. Here we can similarly reconstruct the metric field from $\Xi$ by evaluating its Hochschild field strength

$$
\mathcal{F}_{H}^{\Xi}\left(x^{i}, x^{j}\right)=\Xi\left(x^{i}\right) \star \Xi\left(x^{j}\right)-\Xi\left(x^{i} \star x^{j}\right)=\frac{i \hbar}{2} G^{i j}+o\left(\hbar^{2}\right),
$$

or more directly: $G^{i j}=\frac{2 i}{\hbar}\left(\Xi\left(x^{i} x^{j}\right)-x^{i} x^{j}\right)$. Two more questions come up naturally: When is $\theta=(B+g)_{A}^{-1}$ Poisson? Why is the relevant star product not a quantization of $\tau \equiv B^{-1}$ as in the zero-slope limit? The answer to the second question is of course that this is determined by the open string propagator in the presence of a background $B$ and that happens to have an antisymmetric part given by $\theta^{i j}$ and only in the zero-slope limit this is equal to $B^{-1}$. Nevertheless the two questions turn out to be related - the star products based on $\tau$ and $\theta$ are equivalent provided that the 2 -forms

$$
\boldsymbol{B}=\frac{1}{2}\left(\tau^{-1}\right)_{i j} d x^{i} \wedge d x^{j}, \quad \boldsymbol{\phi}=-\frac{1}{2}(g \tau g)_{i j} d x^{i} \wedge d x^{j}
$$

are closed, $\boldsymbol{\phi}=d \boldsymbol{\alpha}$ for some 1 -form $\boldsymbol{\alpha}$, and $B(t)=B+t \phi$ is nondegenerate for $t \in[0,1]$; the closedness conditions on $\boldsymbol{B}$ and $\boldsymbol{\phi}$ ensure in particular that $\alpha$ and $\theta$ are Poisson. According to Moser's lemma the symplectic structures $B(1)$ and $B(0)$ are related by a change of coordinates generated by the vector field $\chi_{\alpha}=\tau^{i j} \alpha_{j} \partial_{i}$ and, moreover, according to Kontsevich the star products resulting from quantization of $B(0)$ and $B(1)$ are equivalent. Since $B(0)=\tau^{-1}$ and $B(1)=\theta^{-1}$ we have demonstrated our claim. Let us remark that $\boldsymbol{B}$ and $\boldsymbol{\phi}$ are closed if $g$ and $B$ are derived from a Kähler metric, since then $\boldsymbol{B}$ is closed and $B, g B^{-1} g$ are proportional. Instead of using Moser's lemma we could also drop some assumptions and work directly with Poisson structures as in previous sections. We would then require that $\tau$ is Poisson and $g \tau g$ is closed and introduce a 1-parameter deformation $\tau(t), t \in[0,1]$, with

$$
\tau(0)=\tau, \quad \partial_{t} \tau(t)=\tau(t) \cdot(g \tau g) \cdot \tau(t)
$$

and solution

$$
\tau(t)=\tau+t \tau g \tau g \tau+t^{2} \tau g \tau g \tau g \tau g \tau+\ldots=\tau \frac{1}{1-t(g \tau)^{2}} .
$$

This is the antisymmetric part of

$$
\tilde{\theta}(t)=\frac{1}{B+t^{\frac{1}{2}} g} .
$$

The symmetric part is $t^{\frac{1}{2}} G^{i j}(t)=-t^{\frac{1}{2}}[\tau(t) g \tau]^{i j}$ with $G(0)=-B^{-1} g B^{-1}$ and $G(1)=G$, while $\tau(0)=B^{-1}$ and $\tau(1)=\theta$ with $G$ and $\theta$ as given in (133). This suggest that $\phi=-g \tau g$ represents "metric fluctuations" around the background $B$ that can be gauged away by an equivalence transformation that curiously leads to the zero-slope values $G(0), \tau(0)$ of the metric and Poisson structure. 


\section{Seiberg-Witten map for nonabelian gauge fields}

We will now extend the discussion to nonabelian gauge theories, i.e., Lie algebra-valued gauge potentials and gauge fields. We will argue that a Seiberg-Witten map can be explicitly constructed for any gauge group by treating both the space-time noncommutativity and the noncommutativity of the nonabelian gauge group on equal footing. Both structures are obtained from appropriate Poisson structures by deformation quantization. This construction generalizes to fairly arbitrary noncommutative internal spaces.

Let us mention that it is possible to absorb a matrix factor (e.g. GL(n) or $\mathrm{U}(\mathrm{n})$ in the defining representation) directly into the definition of the noncommutative space $\mathcal{A}_{x}$ and then work with the abelian results of the previous sections, however, for other gauge groups it is not a priory clear how to do this consistently. In any case even for GL(n) and $U(n)$ that approach would not give a very detailed description of the nonabelian Seiberg-Witten map.

\subsection{Nonabelian setting}

In this section we shall establish notation and will give a precise definition of the problem that we would like to solve. Consider a manifold "(noncommutative) space-time" with a noncommutative structure provided by a star product that is derived from a Poisson structure $\Theta^{\mu \nu}$. On this space consider a nonabelian gauge theory with gauge group $\mathrm{G}$, field strength $F_{\mu \nu}$, that can be locally expressed as

$$
F_{\mu \nu}=\partial_{\mu} A_{\nu}-\partial_{\nu} A_{\mu}-i\left[A_{\mu}, A_{\nu}\right]
$$

with nonabelian gauge potential $A_{\mu}=A_{\mu b} T^{b}$ where $T^{b} \in \operatorname{Lie}(G)$ are generators with commutation relations $-i\left[T^{a}, T^{b}\right]=C_{c}^{a b} T^{c}$, and nonabelian gauge transformations

$$
\delta_{\Lambda} A_{\mu}=\partial_{\mu} \Lambda+i\left[\Lambda, A_{\mu}\right]
$$

Our main goal is to find a noncommutative gauge potential $\hat{A}=\hat{A}\left(A_{\mu}\right)$ and a noncommutative gauge parameter $\hat{\Lambda}\left(A_{\mu}, \Lambda\right)$ such that a nonabelian gauge transformation $\delta_{\Lambda}$ of $A_{\mu}$ induces a noncommutative gauge transformation $\hat{\delta}_{\hat{\Lambda}}$ of $\hat{A}$ :

$$
\hat{A}\left(A_{\mu}+\delta_{\Lambda} A_{\mu}\right)=\hat{A}\left(A_{\mu}\right)+\hat{\delta}_{\hat{\Lambda}} \hat{A}\left(A_{\mu}\right) .
$$

$\hat{A}\left(A_{\mu}\right)$ should be a universal enveloping algebra-valued formal power series in $\Theta^{\mu \nu}$, starting with $\Theta^{\mu \nu} A_{\nu} \partial_{\mu}$, that contains polynomials of $A_{\mu}$ and it's derivatives. Similarly $\hat{\Lambda}\left(A_{\mu}, \Lambda\right)$ should be a universal enveloping algebra-valued formal power series in $\Theta^{\mu \nu}$, starting with $\Lambda$, that contains polynomials of $A_{\mu}, \Lambda$ and their derivatives. The product in the definition of the noncommutative gauge transformation is a combination of the 
star product on space-time and the matrix product of the $T^{a}$. We expect that it should be possible to find expressions, where the structure constants $C_{c}^{a b}$ do not appear explicitly, except via commutators of the Lie algebra-valued $A_{\mu}, \Lambda$.

A secondary goal is to find a construction that stays as close as possible to the method that we used in the abelian case. There, we used a generalization of Moser's lemma to relate Poisson structures $\boldsymbol{\theta}$ and $\boldsymbol{\theta}^{\prime}$ (and, after quantization, star products $\star$ and $\star^{\prime}$ ). The motivation for this and some of the complications of the nonabelian case can be most easily understood in the special case of invertible, i.e. symplectic, Poisson structures. The inverses of $\theta$ and $\theta^{\prime}$ define closed 2-forms $\boldsymbol{B}$ and $\boldsymbol{B}^{\prime}$ that differ by the addition of a (closed) gauge field $\boldsymbol{f}(62)$. Physically $\boldsymbol{B}^{\prime}$ is the background $\boldsymbol{B}$-field plus fluctuations $\boldsymbol{f}$. In the nonabelian case we would like to keep this picture but with $\boldsymbol{f}$ replaced by the nonabelian field strength $\boldsymbol{F}$ :

$$
\boldsymbol{B}^{\prime}=\boldsymbol{B}+\boldsymbol{F},
$$

where $B=\Theta^{-1}$. The trouble with this is that $d \boldsymbol{F}=-\boldsymbol{A} \wedge \boldsymbol{A} \neq 0$ in the nonabelian case so $\boldsymbol{B}$ and $\boldsymbol{B}^{\prime}$ cannot both be closed 2 -forms, which they should be if we want to interpret their inverses as Poisson structures. Ignoring this, we could then look for a "vector field" $\chi$ that generates a coordinate transformation that relates $\boldsymbol{B}$ and $\boldsymbol{B}^{\prime}$. A natural generalization from the abelian case (69) is

$$
\chi=\Theta^{\mu \nu} A_{\nu} D_{\mu}
$$

where we have replaced the abelian gauge potential by the nonabelian one and have also switched to a covariant derivative $D_{\mu}=\partial_{\mu}+i\left[A_{\mu}, \cdot\right]$. The trouble here is that it is not clear how to act with the matrix-valued $\chi$ on "coordinates". $\chi$ is certainly no vector field; it is not even a derivation. (It's action turns out to involve complete symmetrization over the constituent matrices $T^{a}$.)

The solution to both problems is to consider a larger space that is spanned by the space-time coordinates $x^{\mu}$ and by symbols $t^{a}$ for the generators $T^{a}$ of the Lie Group. $\chi$ is then the projection onto space-time of a true vector field and $\Theta$ and $\Theta^{\prime}$ are the space-time components of true Poisson structures on the enlarged space. We obtain the desired nonabelian noncommutative gauge theory by quantizing both the external and internal part of the enlarged space at the same time. To use the method of the previous sections we need to encode the nonabelian data in an abelian gauge theory on the enlarged space. This program is successful, if the "commutative" nonabelian gauge theory can be recovered at an intermediate step.

\subsection{Abelian data}

Notation: Greek indices $\mu, \nu, \xi, \ldots$ belong to the external space, indices from the beginning of the alphabet $a, b, c, \ldots$ belong to the internal space and indices $i, j$, 
$k, \ldots$ run over the whole space (internal and external). We shall use capital letters $\left(A_{\mu}, F_{\mu \nu}, \Theta^{\mu \nu}\right)$ for things related to the nonabelian theory on the external space and small letters $\left(a_{i}, f_{i j}, \theta^{i j}\right)$ for objects related to the abelian theory on the enlarged space or the internal space $\left(a_{b}, \vartheta^{b c}\right)$.

The $t^{a}$ are commutating coordinate functions on the internal space ("Lie algebra") just like the $x^{\mu}$ are commuting coordinate functions on the external space ("spacetime"). We later recover the Lie algebra in the form of star-commutators on the internal space and the matrices $T^{a}$ by taking a representation of that algebra. The star product on the internal space is a quantization of its natural Poisson structure

$$
\left\{t^{a}, t^{b}\right\}_{\text {Lie }}=C_{c}^{a b} t^{c}=: \vartheta^{a b} .
$$

In the new language

$$
F_{\mu \nu}=\partial_{\mu} A_{\nu}-\partial_{\nu} A_{\mu}+\left\{A_{\mu}, A_{\nu}\right\}_{\text {Lie }}
$$

with $A_{\nu}(x, t)=A_{\nu b}(x) t^{b}$ and

$$
\delta_{\Lambda} A_{\mu}=\partial_{\mu} \Lambda+\left\{A_{\mu}, \Lambda\right\}_{\text {Lie }}
$$

with $\Lambda(x, t)=\Lambda_{b}(x) t^{b}$. ("Lie algebra-valued" translates into "linear in $t$ ".) We equip the enlarged space with a Poisson structure $\theta^{i j}$ which is the direct sum of the external $\Theta^{\mu \nu}$ and internal $\vartheta^{a b}$ Poisson structures

$$
\theta=\left(\begin{array}{l|l}
\Theta & 0 \\
\hline 0 & \vartheta
\end{array}\right)
$$

Only for $t=0$ is the Poisson structure block-diagonal. $\theta(t)$ and in particular $\theta^{\prime}=\theta(1)$ acquire off-diagonal terms through the $t$-evolution

$$
\theta(0)=\theta, \partial_{t} \theta(t)=-\theta(t) f \theta(t) \text {, i.e., } \theta(t)=\theta-t \theta f(t) \theta+t^{2} \theta f(t) \theta f(t) \theta \mp \cdots
$$

generated by an abelian gauge field $f$ that is itself not block-diagonal (but whose internal components $f_{a b}$ are zero as we shall argue below.) The space-time components of $\theta(t)$ can be re-summed in a series in $\Theta$ and we miraculously obtain an expression that looks like the series for $\theta(t)$ but with $f$ replaced by $F_{\mu \nu}(t) \equiv f_{\mu \nu}-t f_{\mu a} \theta^{a b} f_{b \nu}$, which at $t=1$ (and $a_{b}=0$, see below) becomes the nonabelian field strength $F \equiv F(1)=$ $\partial_{\mu} a_{\nu}-\partial_{\nu} a_{\mu}+\left\{a_{\mu}, a_{\nu}\right\}_{\text {Lie }}$ :

$$
\begin{aligned}
\Theta^{\mu \nu}(t) \equiv \theta^{\mu \nu}(t) & =\theta^{\mu \nu}-t \theta^{\mu i} f_{i j} \theta^{j \nu}+t^{2} \theta^{\mu j} f_{j k} \theta^{k l} f_{l m} \theta^{m \nu} \mp \cdots \\
& =\theta^{\mu \nu}-t \theta^{\mu \kappa}\left(f_{\kappa \sigma}-t f_{\kappa a} \theta^{a b} f_{b \sigma}\right) \theta^{\sigma \nu}+\cdots
\end{aligned}
$$

To all orders in $\Theta$ :

$$
\Theta(t)=\Theta-t \Theta F(t) \Theta+t^{2} \Theta F(t) \Theta F(t) \Theta \mp \cdots=\Theta \frac{1}{1+t F(t) \Theta} .
$$


In the case of invertible $\Theta, \Theta^{\prime}$ (with $\Theta^{\prime} \equiv \Theta(1)$ ) we have

$$
\frac{1}{\Theta^{\prime}}=\frac{1}{\Theta}+F
$$

This resembles the relation $B^{\prime}=B+F$ that one would have naively expected, but we should note that $\Theta, \Theta^{\prime}$ are not necessarily Poisson (they are just the space-time components of the Poisson structures $\theta, \theta^{\prime}$ ) and $F$ is not exactly a non-abelian field strength (it is in fact a gauge-invariant expression in abelian gauge fields that coincides with the nonabelian field strength in the special gauge $a_{b}=0$; see next section.) The other components of $\theta(t)$ are computed similarly (again using $f_{a b}=0$ ):

$$
\theta^{\mu b}(t)=-\theta^{b \mu}(t)=-t \Theta^{\mu \nu}(t) f_{\nu a} \vartheta^{a b}, \quad \theta^{a b}(t)=\vartheta^{a b}+t^{2}[\vartheta f \Theta(t) f \vartheta]^{a b} .
$$

$\Theta(t)$ is not the only object that acquires a non-abelian look at $t=1$; this is also the case for Moser's vector field

$$
\mathbf{a}_{\theta^{\prime}}=\left(\theta^{\prime}\right)^{i j} a_{j} \partial_{i}=\left(\Theta^{\prime}\right)^{\mu \nu} \bar{A}_{\nu} \bar{D}_{\mu}+\vartheta^{a b} a_{b} \partial_{a}
$$

where $\bar{A}_{\nu}=a_{\nu}-f_{\nu a} \vartheta^{a b} a_{b}$ and $\bar{D}_{\mu}=\partial_{\mu}-f_{\mu a} \vartheta^{a b} \partial_{b}$ are the first terms in the expansions for the nonabelian gauge potential and covariant derivative, valid around the special gauge $a_{b}=0$ (see next section.)

Now we need to identify appropriate abelian gauge fields and gauge transformations on the enlarged space that upon quantization give the desired nonabelian noncommutative gauge fields and noncommutative gauge transformations. For this we consider the terms of lowest order in $\Theta$ of the Seiberg-Witten condition, where we expect to see a purely nonabelian gauge transformation. Up to this order it is in fact enough to work with the semiclassical condition (79)

$$
A_{a+d \lambda}=A_{a}+\mathbf{d}_{\theta} \tilde{\lambda}+\left\{A_{a}, \tilde{\lambda}\right\} .
$$

Evaluating this on $x^{\mu}$ and collecting terms of order $\Theta$ we get

$$
A_{\mu}(a+d \lambda)=A_{\mu}(a)+\partial_{\mu} \Lambda+\left\{A_{\mu}(a), \Lambda\right\}_{\text {Lie }}
$$

with $A_{\mu}(a)$ and $\Lambda(\lambda, a)$ defined by

$$
A_{a}\left(x^{\nu}\right)=\Theta^{\nu \mu} A_{\mu}(a)+o\left(\Theta^{2}\right), \quad \tilde{\lambda}=\Lambda(\lambda, a)+o(\Theta) .
$$

An abelian gauge transformation $\delta a_{i}=\partial_{i} \lambda$ thus results in a nonabelian gauge transformation of $A_{\mu}$ with gauge parameter $\Lambda$. Since we would like to identify $A_{\mu}$ and $\Lambda$ with the gauge potential and parameter of the ordinary nonabelian gauge theory that we started with, they should both be linear in the coordinates $t^{a}$ of the internal space. Studying gauge transformations and the explicit expression (75), (76) for $A_{a}\left(x^{\mu}\right)$ (see next section) we find that this implies that the internal components of the abelian 
gauge potential are independent of the $t^{a}$, while the external components are linear in the $t^{a}$. This is preserved by abelian gauge transformations with gauge parameters that are linear in the $t^{a}$ :

$$
a_{\mu}=a_{\mu b}(x) t^{b}, \quad a_{b}=a_{b}(x), \quad \lambda=\lambda_{b}(x) t^{b} ; \quad \delta a_{\mu}=i\left(\partial_{\mu} \lambda_{b}\right) t^{b}, \quad \delta a_{b}=i \lambda_{b} .
$$

The gauge invariant characterization of the desired abelian gauge fields is $f_{a b}=0$, $f_{\mu b}=-f_{b \mu}$ independent of the $t^{a}, f_{\mu \nu}$ linear in the $t^{a}$. By a gauge transformation with parameter $-a_{b}(x) t^{b}$ we can always go to a special gauge with vanishing internal gauge potential $a_{b}^{\prime}=0$ (and $a_{\mu}^{\prime}=a_{\mu}-\partial_{\mu}\left(a_{b}\right) t^{b}$.)

We can now apply the method that we developed for the abelian case in the previous sections to obtain the desired nonabelian noncommutative gauge fields in terms of the abelian data (158). These are $\theta$-expanded noncommutative gauge fields that become ordinary nonabelian gauge fields at lowest nontrivial order in $\Theta$ (but all orders in $\vartheta$.) We claim that a re-summation of the $\theta$-series gives in fact $\Theta$-expanded noncommutative gauge fields in terms of nonabelian gauge fields. This is a much stronger statement and can be checked by inspection using the special gauge $a_{b}=0$. A rigorous formal proof is however missing.

\subsection{Mini Seiberg-Witten map}

Nonabelian gauge theory is of course also a type of noncommutative gauge theory and one may thus wonder whether a Seiberg-Witten map exists from abelian to nonabelian gauge fields. Computing $A_{\mu}(a)$ and $\Lambda(\lambda, a)$ (157) using the results from the previous sections does in fact provide such maps:

$$
\begin{aligned}
& A_{\mu}(a)=\left(e^{\mathbf{a}_{\vartheta}}\right)\left(a_{\mu}-\partial_{\mu} \alpha\right)+\left(\frac{e^{\mathbf{a}_{\vartheta}}-1}{\mathbf{a}_{\vartheta}}\right)\left(\partial_{\mu} \alpha\right), \\
& \Lambda(\lambda, a)=\left(\frac{e^{\mathbf{a}_{\vartheta}}-1}{\mathbf{a}_{\vartheta}}\right)(\lambda)
\end{aligned}
$$

with $\alpha(x, t)=a_{b}(x) t^{b}$, the parameter of the gauge transformation that gives $a_{b}=\partial_{b} \alpha$ starting from the special gauge $a_{b}=0$. Note that $\mathbf{a}_{\vartheta}=\vartheta^{a b} a_{b} \partial_{a}=\{\cdot, \alpha\}_{\text {Lie. }}$. In components

$$
\begin{aligned}
& A_{\mu}(a)=\sum_{n=0}^{\infty} \frac{1}{(n+1) !} t^{a}\left(M^{n}\right)_{a}^{b}\left(a_{\mu b}-n f_{\mu b}\right), \\
& \Lambda(\lambda, a)=\sum_{n=0}^{\infty} \frac{1}{(n+1) !} t^{a}\left(M^{n}\right)_{a}^{b} \lambda_{b},
\end{aligned}
$$


with the matrix $M_{a}^{b}=C_{a}^{b c} a_{c}$ and $a_{\mu b} t^{b}=a_{\mu}, \lambda_{b} t^{b}=\lambda$. Under an abelian gauge transformation $\delta_{\lambda} a_{i}=\partial_{i} \lambda$,

$$
\delta_{\lambda} A_{\mu}(a)=\partial_{\mu} \Lambda(\lambda, a)+\left\{A_{\mu}(a), \Lambda(\lambda, a)\right\}_{\text {Lie }} .
$$

In the special gauge of vanishing internal gauge potential $a_{b}=0$ the maps becomes simply $A_{\mu}(a)=a_{\mu}, \Lambda(\lambda, a)=\lambda$.

\section{Acknowledgments}

We would like to thank P. Aschieri, J. Madore, S. Schraml and A. Sitarz for helpful discussions.

\section{A Brackets, evolution and parameters}

\section{A.1 Schouten-Nijenhuis bracket $\mathrm{H}^{\mathrm{H}}$}

The Schouten-Nijenhuis bracket of two polyvector fields is defined by

$$
\begin{aligned}
{\left[\xi_{1} \wedge \ldots \wedge \xi_{k}, \eta_{1} \wedge\right.} & \left.\ldots \wedge \eta_{l}\right]_{\mathrm{S}} \\
& =\sum_{i, j}(-)^{i+j}\left[\xi_{i}, \eta_{j}\right] \wedge \xi_{1} \wedge \ldots \wedge \hat{\xi}_{i} \wedge \ldots \wedge \hat{\eta}_{j} \wedge \ldots \wedge \eta_{l}, \\
{\left[f, \xi_{1} \wedge \ldots \wedge \xi_{k}\right]_{\mathrm{S}} } & =\sum_{i}(-)^{i-1} \xi_{i}(f) \xi_{1} \wedge \ldots \wedge \hat{\xi}_{i} \wedge \ldots \wedge \xi_{k},
\end{aligned}
$$

if all $\xi$ 's and $\eta$ 's are vector fields and $f$ is a function. The hat marks omitted vector fields. A Poison tensor is a bivector field $\theta=\frac{1}{2} \theta^{i j} \partial_{i} \wedge \partial_{j}$ that satisfies the Jacobi identity

$$
0=[\theta, \theta]_{\mathrm{S}} \equiv \frac{1}{3}\left(\theta^{i l} \partial_{l}\left(\theta^{j k}\right)+\theta^{j l} \partial_{l}\left(\theta^{k i}\right)+\theta^{k l} \partial_{l}\left(\theta^{i j}\right)\right) \partial_{i} \wedge \partial_{j} \wedge \partial_{k}
$$

In terms of the coboundary operator

$$
\mathbf{d}_{\theta}=-[\cdot, \theta]_{\mathrm{S}},
$$

this can also be expressed as $\mathbf{d}_{\theta} \theta=0$ or $\mathbf{d}_{\theta}^{2}=0$.

\footnotetext{
${ }^{13} \mathrm{~A}$ good reference for the material in this section and the next is 47 .
} 


\section{A.2 Gerstenhaber bracket}

The Gerstenhaber bracket is given by

$$
\left[\mathcal{C}_{1}, \mathcal{C}_{2}\right]_{\mathrm{G}}=\mathcal{C}_{1} \circ \mathcal{C}_{2}-(-)^{\left(p_{2}+1\right)\left(p_{1}+1\right)} \mathcal{C}_{2} \circ \mathcal{C}_{1}
$$

where composition $\circ$ for $\mathcal{C}_{1} \in C^{p_{1}}$ and $\mathcal{C}_{2} \in C^{p_{2}}$ is defined as

$$
\begin{aligned}
\left(\mathcal{C}_{1} \circ \mathcal{C}_{2}\right)\left(f_{1}, f_{2}, \ldots, f_{p_{1}+p_{2}-1}\right)=\mathcal{C}_{1}\left(\mathcal{C}_{2}\left(f_{1}, \ldots, f_{p_{2}}\right), f_{p_{2}+1}, \ldots, f_{p_{2}+p_{1}-1}\right) \\
\quad-(-)^{p_{2}} \mathcal{C}_{1}\left(f_{1}, \mathcal{C}_{2}\left(f_{2}, \ldots, f_{p_{2}+1}\right), f_{p_{2}+2}, \ldots, f_{p_{2}+p_{1}-1}\right) \\
\quad \pm \ldots+(-)^{\left(p_{2}+1\right)\left(p_{1}+1\right)} \mathcal{C}_{1}\left(f_{1}, \ldots, f_{p_{1}-1}, \mathcal{C}_{2}\left(f_{p_{1}}, \ldots, f_{p_{1}+p_{2}-1}\right)\right)
\end{aligned}
$$

$C^{p}$ may be either $\operatorname{Hom}_{k}\left(\mathcal{A}_{x}^{\otimes p}, \mathcal{A}_{x}\right)$ or the space of $p$-differential operators $D_{\text {poly }}^{p}$. In analogy to (A.2) we can express the associativity of a product $\star \in C^{2}$ as

$$
[\star, \star]_{\mathrm{G}}=0, \quad[\star, \star]_{\mathrm{G}}(f, g, h) \equiv 2((f \star g) \star h-f \star(g \star h)) .
$$

In terms of the coboundary operator (see also (29))

$$
\mathbf{d}_{\star}: C^{p} \rightarrow C^{p+1}, \quad \mathbf{d}_{\star} \mathcal{C}=-[\mathcal{C}, \star]_{\mathrm{G}},
$$

this can also be written as $\mathbf{d}_{\star} \star=0$ or $\mathbf{d}_{\star}^{2}=0$.

\section{A.3 t-evolution}

Consider a $t$-dependent function $f(t)$ whose $t$-evolution is governed by

$$
\left(\partial_{t}+A(t)\right) f(t)=0
$$

where $A(t)$ is an operator (vector field or differential operator of arbitrary degree) whose $t$-dependence is given. We are interested to relate $f(1)$ to $f(0)$. There is a simple way to integrate (A.8) without having to resort to $t$-ordered exponentials: By Taylor expansion

$$
e^{-\partial_{t}} f(t)=f(t-1)
$$

Due to $\left(\right.$ A.8) we can insert $\exp \left(\partial_{t}+A(t)\right)$ without changing anything

$$
e^{-\partial_{t}} e^{\partial_{t}+A(t)} f(t)=f(t-1) \text {. }
$$

The trick hereby is that due to the Baker-Campbell-Hausdorff formula all $\partial_{t}$ are saturated in the product of the exponentials; there are no free $t$-derivatives acting on $f(t)$, so we can evaluate at $t=1$ and get

$$
\left.e^{-\partial_{t}} e^{\partial_{t}+A(t)}\right|_{t=1} f(1)=f(0)
$$

or, slightly rearranged

$$
\left.e^{\partial_{t}+A(t)} e^{-\partial_{t}}\right|_{t=0} f(1)=f(0) \text {. }
$$

The first few terms in the expansion of the exponentials are $1+A+\frac{1}{2}\left(A^{2}+\dot{A}\right)+\cdots$. 


\section{A.4 Semi-classical and quantum gauge parameters}

Let $A$ and $B$ be two operators (vector fields or differential operators) and

$$
B_{0} \equiv B, \quad B_{n+1}=\left[A, B_{n}\right]
$$

then

$$
e^{A+\epsilon B}-e^{A}=\epsilon \sum_{n=0}^{\infty} \frac{B_{n}}{(n+1) !} e^{A}+o\left(\epsilon^{2}\right) .
$$

Semi-classical: We would like to proof (75) and (80)

$$
\rho_{a+d \lambda}^{*}-\rho_{a}^{*}=\left(\mathbf{d}_{\theta} \tilde{\lambda}\right) \circ \rho_{a}^{*}+o\left(\lambda^{2}\right), \quad \tilde{\lambda}(\lambda, a)=\sum_{n=0}^{\infty} \frac{\left.\left(\mathbf{a}_{\theta_{t}}+\partial_{t}\right)^{n}(\lambda)\right|_{t=0}}{(n+1) !} .
$$

It is helpful to first evaluate

$$
\left[\partial_{t}+\mathbf{a}_{\theta_{t}}, \mathbf{d}_{\theta_{t}} \lambda\right]=\mathbf{d}_{\theta_{t}}\left[\left(\mathbf{a}_{\theta_{t}}+\partial_{t}\right)(\lambda)\right]
$$

(Note that both $\mathbf{d}_{\theta_{t}} \lambda$ and $\mathbf{d}_{\theta_{t}} \mathbf{a}_{\theta_{t}}(\lambda)$ are Hamiltonian vector fields.)

Proof: (we suppress the $t$-subscripts and the explicit $t$-dependence of $\lambda$ )

$$
\begin{aligned}
{\left[\partial_{t}+\mathbf{a}_{\theta}, \mathbf{d}_{\theta} \lambda\right](f) } & =\partial_{t}(\{f, \lambda\})+\mathbf{a}_{\theta}(\{f, \lambda\})-\left\{\mathbf{a}_{\theta}(f), \lambda\right\} \\
& =\mathbf{f}_{\theta}(f, \lambda)-\left(\mathbf{d}_{\theta} \mathbf{a}_{\theta}\right)(f, \lambda)+\left\{f, \mathbf{a}_{\theta}(\lambda)\right\} \\
& =\mathbf{d}_{\theta}\left(\mathbf{a}_{\theta}(\lambda)\right)(f) .
\end{aligned}
$$

We have repeatedly used the definition of $\mathbf{d}_{\theta}$ (68) and, in the last step, (70). Now we can use (A.14) to evaluate

$$
\begin{aligned}
\rho_{a+d \lambda}^{*}-\rho_{a}^{*} & =\left.\left(e^{\partial_{t}+\mathbf{a}_{\theta_{t}}+\mathbf{d}_{\theta_{t}} \lambda}-e^{\partial_{t}+\mathbf{a}_{\theta_{t}}}\right) e^{-\partial_{t}}\right|_{t=0} \\
& =\left.\sum_{n=0}^{\infty} \frac{\mathbf{d}_{\theta_{t}}\left(\mathbf{a}_{\theta_{t}}+\partial_{t}\right)^{n}(\lambda)}{(n+1) !} e^{\partial_{t}+\mathbf{a}_{\theta_{t}}} e^{-\partial_{t}}\right|_{t=0}+o\left(\lambda^{2}\right)
\end{aligned}
$$

Quantum: We would like to proof (106) and (108)

$$
\mathcal{D}_{a+d \lambda}-\mathcal{D}_{a}=\left(\frac{1}{i \hbar} \mathbf{d}_{\star} \Lambda\right) \circ \mathcal{D}_{a}+o\left(\lambda^{2}\right), \quad \Lambda(\lambda, a)=\sum_{n=0}^{\infty} \frac{\left.\left(\mathbf{a}_{\star t}+\partial_{t}\right)^{n}(\hat{\lambda})\right|_{t=0}}{(n+1) !}
$$

First we evaluate

$$
\left[\partial_{t}+\mathbf{a}_{\star t}, \mathbf{d}_{\star_{t}} \hat{\lambda}\right]=\mathbf{d}_{\star_{t}}\left[\left(\mathbf{a}_{\star_{t}}+\partial_{t}\right)(\hat{\lambda})\right] .
$$


(Note that both $\mathbf{d}_{\star t} \hat{\lambda}$ and $\mathbf{d}_{\star t} \mathbf{a}_{\star t}(\hat{\lambda})$ are inner derivations. Also note that the proof of this equation is in principle much harder than its semi-classical counterpart, since we are now dealing with differential operators of arbitrary degree.)

Proof: (we suppress the $t$-subscripts and the explicit $t$-dependence of $\lambda$ )

$$
\begin{aligned}
{\left[\partial_{t}+\mathbf{a}_{\star}, \mathbf{d}_{\star} \hat{\lambda}\right](f) } & =\partial_{t}[f, \hat{\lambda}]_{\star}+\mathbf{a}_{\star}\left(\mathbf{d}_{\star}(\hat{\lambda}) f\right)-\mathbf{d}_{\star}(\hat{\lambda})\left(\mathbf{a}_{\star}(f)\right) \\
& =\mathbf{f}_{\star}(f, \hat{\lambda})-\mathbf{f}_{\star}(\hat{\lambda}, f)+\mathbf{a}_{\star}\left([f, \hat{\lambda}]_{\star}\right)-\left[\mathbf{a}_{\star}(f), \hat{\lambda}\right]_{\star} \\
& =\mathbf{f}_{\star}(f, \hat{\lambda})-\mathbf{f}_{\star}(\hat{\lambda}, f)-\left(\mathbf{d}_{\star} \mathbf{a}_{\star}\right)(f, \hat{\lambda})+\left[f, \mathbf{a}_{\star} \hat{\lambda}\right]_{\star}+\left(\mathbf{d}_{\star} \mathbf{a}_{\star}\right)(\hat{\lambda}, f) \\
& =\mathbf{d}_{\star}\left(\mathbf{a}_{\star}(\hat{\lambda})\right)(f) .
\end{aligned}
$$

The desired result (A.19) follows now from (A.14) as in the semi-classical case.

\section{References}

[1] J. Madore, S. Schraml, P. Schupp and J. Wess, "Gauge theory on noncommutative spaces," Eur. Phys. J. C 16, 161 (2000) hep-th/0001203].

[2] C. G. Callan, C. Lovelace, C. R. Nappi, S. A. Yost, String loop corrections to beta functions, Nucl. Phys. B288, 525 (1987); A. Abouelsaood, C. G. Callan, C. R. Nappi, S. A. Yost, Open Strings in Background Gauge Fields, Nucl. Phys. B280, 599 (1987).

[3] C. Chu and P. Ho, "Noncommutative open string and D-brane," Nucl. Phys. B 550, 151 (1999) hep-th/9812219]; "Constrained quantization of open string in background B field and noncommutative D-brane," Nucl. Phys. B 568, 447 (2000) hep-th/9906192].

[4] V. Schomerus, "D-branes and deformation quantization," JHEP9906, 030 (1999) hep-th/9903205].

[5] A. Connes, M. R. Douglas and A. Schwarz, "Noncommutative geometry and matrix theory: Compactification on tori," JHEP9802, 003 (1998) [hepth/9711162.

[6] M. R. Douglas and C. Hull, "D-branes and the noncommutative torus," JHEP9802, 008 (1998) hep-th/9711165.

[7] B. Morariu and B. Zumino, "Super Yang-Mills on the noncommutative torus," in: Relativity, Particle Physics and Cosmology, World Scientific, Singapore, 1998, hep-th/9807198. 
[8] W. I. Taylor, "D-brane field theory on compact spaces," Phys. Lett. B 394, 283 (1997) hep-th/9611042.

[9] N. Seiberg and E. Witten, "String theory and noncommutative geometry," JHEP9909, 032 (1999) hep-th/9908142.

[10] O. Andreev and H. Dorn, "On open string sigma-model and noncommutative gauge fields," Phys. Lett. B 476, 402 (2000) hep-th/9912070.

[11] B. Jurco and P. Schupp, "Noncommutative Yang-Mills from equivalence of star products," Eur. Phys. J. C 14, 367 (2000) hep-th/0001032.

[12] B. Jurco, P. Schupp and J. Wess, "Noncommutative gauge theory for Poisson manifolds," Nucl. Phys. B 584, 784 (2000) hep-th/0005005.

[13] M. Kontsevich, "Deformation quantization of Poisson manifolds, I," q-alg/9709040.

[14] L. Cornalba and R. Schiappa, "Nonassociative star product deformations for D-brane worldvolumes in curved backgrounds," hep-th/0101219.

[15] B. Jurco, P. Schupp and J. Wess, "Nonabelian noncommutative gauge fields and Seiberg-Witten map," hep-th/0012225.

[16] B. Jurco, S. Schraml, P. Schupp and J. Wess, "Enveloping algebra valued gauge transformations for non-Abelian gauge groups on non-commutative spaces," Eur. Phys. J. C 17, 521 (2000) hep-th/0006246].

[17] F. Bayen, M. Flato, C. Fronsdal, A. Lichnerowicz, D. Sternheimer, Deformation theory and quantization. I. Deformations of symplectic structures, Ann. Physics 111, 61 (1978).

[18] D. Sternheimer, "Deformation quantization: Twenty years after," math.qa/9809056.

[19] A. S. Cattaneo and G. Felder, "A path integral approach to the Kontsevich quantization formula," Commun. Math. Phys. 212, 591 (2000) math.qa/9902090.

[20] P. Schaller and T. Strobl, "Poisson structure induced (topological) field theories," Mod. Phys. Lett. A 9, 3129 (1994) hep-th/9405110.

[21] N. Ikeda, "Two-dimensional gravity and nonlinear gauge theory," Annals Phys. 235, 435 (1994) hep-th/9312059. 
[22] P. Schaller and T. Strobl, "Poisson sigma models: A generalization of 2-d gravity Yang-Mills systems," hep-th/9411163; "Introduction to Poisson sigmamodels," hep-th/9507020.

[23] A. Y. Alekseev, P. Schaller and T. Strobl, "The Topological G/G WZW model in the generalized momentum representation," Phys. Rev. D 52, 7146 (1995) hep-th/9505012.

[24] L. Bonora and M. Salizzoni, "Renormalization of noncommutative U(N) gauge theories," hep-th/0011088.

[25] A. Armoni, "Comments on Perturbative Dynamics of Non-Commutative YangMills Theory," Nucl. Phys. B593, 229 (2001) hep-th/0005208.

[26] A. Armoni, R. Minasian and S. Theisen, "On non-commutative $\mathrm{N}=2$ super Yang-Mills," hep-th/0102007.

[27] A. A. Bichl, J. M. Grimstrup, L. Popp, M. Schweda and R. Wulkenhaar, "Perturbative analysis of the Seiberg-Witten map," hep-th/0102044.

[28] A. A. Bichl, J. M. Grimstrup, L. Popp, M. Schweda and R. Wulkenhaar, "Deformed QED via Seiberg-Witten Map," hep-th/0102103.

[29] A. Connes, "Noncommutative geometry," Academic Press, 1994; "Noncommutative geometry: Year 2000," math.qa/0011193.

[30] J. Moser, On the volume elements on a manifold, Trans. Amer. Math. Soc. 120, 286 (1965).

[31] K. Okuyama, "A path integral representation of the map between commutative and noncommutative gauge fields," JHEP0003, 016 (2000) hep-th/9910138.

[32] N. Seiberg, "A note on background independence in noncommutative gauge theories, matrix model and tachyon condensation," JHEP0009, 003 (2000) hep-th/0008013.

[33] L. Cornalba, "D-brane physics and noncommutative Yang-Mills theory," Adv. Theor. Math. Phys. (in press) [hep-th/9909081].

[34] L. Cornalba, "Corrections to the Abelian Born-Infeld action arising from noncommutative geometry," JHEP0009, 017 (2000) hep-th/9912293.

[35] N. Ishibashi, "A relation between commutative and noncommutative descriptions of D-branes," hep-th/9909176. 
[36] L. Cornalba, "On the general structure of the non-Abelian Born-Infeld action," hep-th/0006018.

[37] D. Arnal, D. Manchon, M. Masmoudi, Choix des signes pour la formalite de M. Kontsevich, math.QA/0003003.

[38] D. Manchon, Poisson bracket, deformed bracket and gauge group actions in Kontsevich deformation quantization, math.QA/0003004.

[39] P. Aschieri, private communication.

[40] S. R. Das, S.-J. Rey, "Open Wilson lines in noncommutative gauge theory and tomography of holographic dual supergravity," Nucl. Phys. B590, 453 (2000) hep-th/0008042.

[41] T. Mehen and M. B. Wise, "Generalized *-products, Wilson lines and the solution of the Seiberg-Witten equations," JHEP0012, 008 (2000) [hepth/0010204.

[42] H. Liu, $\star-T r e k ~ I I: \star_{n}$ Operations, Open Wilson Lines and the Seiberg-Witten Map, hep-th/0011125.

[43] S. R. Das, S. P. Trivedi, "Supergravity couplings to noncommutative branes, open wilson lines and generalized star products," hep-th/0011131.

[44] K. Okuyama, "Comments on open Wilson lines and generalized star products," hep-th/0101177.

[45] J. Madore, S. Schraml, P. Schupp and J. Wess, "External fields as intrinsic geometry," Eur. Phys. J. C (online) DOI:10.1007/s100520100566 thepth/0009230.

[46] T. Asakawa and I. Kishimoto, "Noncommutative gauge theories from deformation quantization," Nucl. Phys. B 591, 611 (2000) hep-th/0002138].

[47] A. Cannas du Silvia, A. Weinstein, Geometric Models for Noncommutative Algebras, AMS (1999). 